\title{
An extensive experimental survey of regression methods
}

\author{
M. Fernández-Delgado, M.S. Sirsat, E. Cernadas, S. Alawadi, S. Barro and \\ M. Febrero-Bande
}

Version: accepted article

\section{How to cite:}

M. Fernández-Delgado, M.S. Sirsat, E. Cernadas, S. Alawadi, S. Barro and M. Febrero-Bande (2019) An extensive experimental survey of regression methods. Neural Networks, 111, 11 - 34.

Doi: https://doi.org/10.1016/j.neunet.2018.12.010

\section{Copyright information:}

(C) 2018 Elsevier Ltd. This manuscript version is made available under the CC-BY-NC-ND 4.0 license 


\title{
An extensive experimental survey of regression methods
}

\author{
M. Fernández-Delgado ${ }^{1, *}$, M.S. Sirsat ${ }^{1}$, E. Cernadas ${ }^{1}$, S. Alawadi ${ }^{1}$, S. \\ Barro $^{1}$, M. Febrero-Bande ${ }^{2}$
}

\begin{abstract}
Regression is a very relevant problem in machine learning, with many different available approaches. The current work presents a comparison of a large collection composed by 77 popular regression models which belong to 19 families: linear and generalized linear models, generalized additive models, least squares, projection methods, LASSO and ridge regression, Bayesian models, Gaussian processes, quantile regression, nearest neighbors, regression trees and rules, random forests, bagging and boosting, neural networks, deep learning and support vector regression. These methods are evaluated using all the regression datasets of the UCI machine learning repository (83 datasets), with some exceptions due to technical reasons. The experimental work identifies several outstanding regression models: the M5 rule-based model with corrections based on nearest neighbors (cubist), the gradient
\end{abstract}

\footnotetext{
${ }^{*}$ M. Fernández-Delgado

Email address: manuel.fernandez.delgado@usc.es (M. Fernández-Delgado ) $U R L$ :

https://citius.usc.es/equipo/persoal-adscrito/manuel-fernandez-delgado (M. Fernández-Delgado )

${ }^{1}$ Centro Singular de Investigación en Tecnoloxías da Información da USC (CiTIUS), University of Santiago de Compostela, Campus Vida, 15782, Santiago de Compostela, Spain.

${ }^{2}$ Dept. of Statistics, Mathematical Analysis and Optimization, University of Santiago de Compostela, Campus Vida, 15782, Santiago de Compostela, Spain.
} 
boosted machine (gbm), the boosting ensemble of regression trees (bstTree) and the M5 regression tree. Cubist achieves the best squared correlation $\left(R^{2}\right)$ in $15.7 \%$ of datasets being very near to it, with difference below 0.2 for $89.1 \%$ of datasets, and the median of these differences over the dataset collection is very low (0.0192), compared e.g. to the classical linear regression (0.150). However, cubist is slow and fails in several large datasets, while other similar regression models as M5 never fail and its difference to the best $R^{2}$ is below 0.2 for $92.8 \%$ of datasets. Other well-performing regresors are the committee of neural networks (avNNet), extremely randomized regression trees (extraTrees, which achieves the best $R^{2}$ in $33.7 \%$ of datasets), random forest ( $r f$ ) and $\varepsilon$-support vector regression (svr), but they are slower and fail in several datasets. The fastest regression model is least angle regression lars, which is 70 and 2,115 times faster than M5 and cubist, respectively. The model which requires least memory is non-negative least squares (nnls), about 2 GB, similarly to cubist, while M5 requires about 8 GB. For $97.6 \%$ of datasets there is a regression model among the 10 bests which is very near (difference below 0.1 ) to the best $R^{2}$, which increases to $100 \%$ allowing differences of 0.2 . Therefore, provided that our dataset and model collection are representative enough, the main conclusion of this study is that, for a new regression problem, some model in our top-10 should achieve $R^{2}$ near to the best attainable for that problem.

Keywords: Regression, UCI machine learning repository, cubist, M5, gradient boosted machine, extremely randomized regression tree, support vector regression penalized linear regression. 


\section{Introduction}

The objective of this paper is to provide a "road map" for researchers who want to solve regression problems and need to know how well work the currently available regression methods. In machine learning, regression methods are designed to predict continuous numeric outputs where an order relation is defined. Regression has been widely studied from the statistics field, which provides different approaches to this problem: linear and generalized linear regression, least and partial least squares regression (LS and PLS), least absolute shrinkage and selection operator (LASSO) and ridge regression, multivariate adaptive regression splines (MARS), least angle regression (LARS), among others. Furthermore, several methods arising from the field of machine learning were designed to be universal function aproximators, so they can be applied both for classification and regression: neural networks, support vector machines, regression trees and rules, bagging and boosting ensembles, random forests and others. The current work develops an empirical quantitative comparison of a very large collection of regression techniques which is intended to provide the reader: 1) a list of the currently available regression models, grouped by families of related methods; 2) a brief description and list of references about each approach, alongside with technical details about its execution such as software implementation, list of tunable hyperparameters and recommended values; 3) a ranking of the available models according to its performance and speed, identifying the best performing approach and the performance level which can be expected for it; and 4) the code to run all the regression models considered in this study for 
any regression problem 3 . In this comparison, we use the whole collection of regression datasets provided of the UCI machine learning repository (excepting some datasets excluded by technical reasons), which a large collection of regression problems, and it should allow to develop a realistic and significant evaluation of the regression methods. As we explained in a previous paper comparing classifiers [1], provided that the size of the model collection used in the current comparison is large enough, we can assume that the best performance, measured in terms of squared correlation $\left(R^{2}\right)$, achieved by some regression model for each dataset (denoted as $R_{\text {best }}^{2}$ ) is the highest attainable performance for that dataset. For a model which achieves a given $R^{2}$ in that dataset, the difference $\Delta=R_{\text {best }}^{2}-R^{2}$, averaged over the dataset collection, can be used as an estimation of the expected $\Delta$ for that model and a new dataset $D$, not included in the collection. For the best model $X$ on the current comparison, it is expected that $\Delta \gtrsim 0$, i.e., the $R^{2}$ achieved by $X$ should not be too far from $R_{\text {best }}$ in average over the data collection. Thus, although by the No-Free-Lunch theorem [2] we can not guarantee that $X$ will be the best model for $D$, we can expect that $X$ will achieve $R^{2}>R_{\text {best }}^{2}-\Delta$, so that $X$ will not be very far from $R_{\text {best }}^{2}$ for dataset $D$. Consequently, the current paper may be useful for researchers who want to know how far a given model (e.g. the best model $X$ ) will be from the best available performance (which is, of course, unknown) for a new dataset. On the other hand, in general the best models in the current comparison achieve the best, or very near to the best, performances for most datasets in the collection. Therefore, although

\footnotetext{
${ }^{3}$ https://nextcloud.citius.usc.es/index.php/s/Yb8LZQQFrgckjFk (visited December $14,2018)$.
} 
$X$ will not be the best regression model for a new dataset $D$, we can expect that some of the best models in our comparison will achieve the best $R^{2}$. Thus, the current comparison may be also useful to provide to the reader a reduced list (e.g., the 10 best performing models of the collection) which is expected to include the one which provides the highest available performance for a new dataset $D$.

The section 2 describes the materials and methods used for this comparison, which include the list of datasets and regression methods, grouped by families. The description of regression models and issues related to their execution (software implementation, number of tunable hyperparameters and their values) are included in Appendix B. The section 3 reports the results of the experimental work and discusses them globally, by families of regression models and by datasets, best model for each dataset, elapsed time and memory. Finally, the section 4 compiles the conclusions of this study.

\section{Materials and methods}

This section describes the scope of the current work, defined by the collection of datasets used in this comparison (subsection 2.1) and by the regression methods that will be compared (subsection 2.2).

\begin{tabular}{|l|l|c|c|}
\hline Original UCI name & Datasets & \#patterns & \#inputs \\
\hline \hline 3D Road network & 3Droad & 434,874 & $4 / 3$ \\
\hline Airfoil self-noise & airfoil & 1,503 & 5 \\
\hline \multirow{3}{*}{ Air quality } & $\begin{array}{l}\text { air-quality-CO, air-quality-NMHC } \\
\text { air-quality-NO2, air-quality-NOx } \\
\text { air-quality-O3 }\end{array}$ & 1,230 & 8 \\
\hline Appliances energy prediction & appliances-energy & 19,735 & $28 / 26$ \\
\hline Auto MPG & auto-MPG & 398 & $8 / 23$ \\
\hline Automobile & automobile & 205 & $26 / 66$ \\
\hline
\end{tabular}


Table 2 - Continued from previous page.

\begin{tabular}{|c|c|c|c|}
\hline Original UCI name & Datasets & \#patterns & \#inputs \\
\hline Beijing PM2.5 & beijing-pm25 & 41,758 & 12 \\
\hline \multirow{2}{*}{ Bike sharing } & bike-day & 731 & $13 / 30$ \\
\hline & bike-hour & 17,379 & $14 / 42$ \\
\hline Blog feedback & blog-feedback & 60,021 & $280 / 13$ \\
\hline Buzz in social media & buzz-twitter & 583,250 & 77 \\
\hline Combined cycle power plant & combined-cycle & 9,568 & 4 \\
\hline Communities \& crime & com-crime & 1,994 & 122 \\
\hline Communities \& crime unnormalized & com-crime-unnorm & 2,215 & $124 / 126$ \\
\hline Computer hardware & com-hd & 209 & 7 \\
\hline Concrete compressive strength & compress-stren & 1,030 & 8 \\
\hline \multirow{2}{*}{ Concrete slump test } & slump & \multirow{2}{*}{103} & 9 \\
\hline & slump-comp, slump-flow & & 7 \\
\hline $\begin{array}{l}\text { Condition based maintenance of naval } \\
\text { propulsion plants }\end{array}$ & cond-turbine & 11,934 & 13 \\
\hline $\begin{array}{l}\text { Conventional and Social Media Movies } \\
14 / 15\end{array}$ & $\operatorname{csm} 1415$ & 231 & $12 / 11$ \\
\hline Cuff-less blood pressure estimation & cuff-less & 61,000 & $3 / 2$ \\
\hline Daily Demand Forecasting Orders & daily-demand & 60 & $13 / 12$ \\
\hline $\begin{array}{l}\text { Dynamic features of VirusShare Exe- } \\
\text { cutables }\end{array}$ & dynamic-features & 107,856 & $482 / 265$ \\
\hline Energy efficiency & energy-cool, energy-heat & 768 & $8 / 7$ \\
\hline Facebook comment volume & facebook-comment & 40,949 & $54 / 48$ \\
\hline Facebook metrics & facebook-metrics & 500 & 19 \\
\hline Forestfires & forestfires & 517 & $12 / 39$ \\
\hline \multirow{2}{*}{$\begin{array}{l}\text { Gas sensor array under dynamic } \\
\text { gas mixtures }\end{array}$} & gas-dynamic-CO & \multirow{2}{*}{58} & \multirow{2}{*}{$438 / 57$} \\
\hline & gas-dynamic-methane & & \\
\hline \multirow{2}{*}{ Geographical original of music } & geo-lat, geo-long & \multirow{2}{*}{1,059} & $116 / 72$ \\
\hline & geo-music-lat, geo-music-long & & 68 \\
\hline GPS trajectories & gps-trajectory & 163 & 10 \\
\hline Greenhouse gas observing network & greenhouse-net & 955,167 & 15 \\
\hline Housing & housing & 452 & 13 \\
\hline $\begin{array}{l}\text { Individual household electric power } \\
\text { consumption }\end{array}$ & household-consume & $2,049,280$ & $6 / 5$ \\
\hline Istanbul stock exchange & stock-exchange & 536 & 8 \\
\hline $\begin{array}{l}\text { KEGG metabolic reaction network } \\
\text { (undirected) }\end{array}$ & KEGG-reaction & 65,554 & $27 / 25$ \\
\hline $\begin{array}{l}\text { KEGG metabolic relation network (di- } \\
\text { rected) }\end{array}$ & KEGG-relation & 54,413 & $22 / 17$ \\
\hline Online news popularity & online-news & 39,644 & $59 / 55$ \\
\hline $\begin{array}{l}\text { Online video characteristics and } \\
\text { transcoding time dataset }\end{array}$ & video-transcode & 68,784 & $20 / 8$ \\
\hline
\end{tabular}


Table 2 - Continued from previous page.

\begin{tabular}{|c|c|c|c|}
\hline Original UCI name & Datasets & \#patterns & \#inputs \\
\hline $\begin{array}{l}\text { Parkinson speech dataset with multi- } \\
\text { ple types of sound recordings }\end{array}$ & park-speech & 1,040 & 26 \\
\hline Parkinson's telemonitoring & $\begin{array}{l}\text { park-motor-UPDRS, } \quad \text { park-total- } \\
\text { UPDRS }\end{array}$ & 5,875 & 16 \\
\hline \multirow{16}{*}{ PM2.5 Data 5 Chinese Cities } & pm25-beijing-dongsi & 24,237 & \\
\hline & pm25-beijing-dongsihuan & 20,166 & \multirow{15}{*}{13} \\
\hline & pm25-beijing-nongzhanguan & 24,137 & \\
\hline & pm25-beijing-us-post & 49,579 & \\
\hline & pm25-chengdu-caotangsi & 22,997 & \\
\hline & pm25-chengdu-shahepu & 23,142 & \\
\hline & pm25-chengdu-us-post & 27,368 & \\
\hline & pm25-guangzhou-city-station & 32,351 & \\
\hline & pm25-guangzhou-5th-middle-school & 21,095 & \\
\hline & pm25-guangzhou-us-post & 32,351 & \\
\hline & pm25-shanghai-jingan & 22,099 & \\
\hline & pm25-shanghai-us-post & 31,180 & \\
\hline & pm25-shanghai-xuhui & 23,128 & \\
\hline & pm25-shenyang-taiyuanji & 22,992 & \\
\hline & pm25-shenyang-us-post & 20,452 & \\
\hline & pm25-shenyang-xiaoheyan & 23,202 & \\
\hline $\begin{array}{l}\text { Physicochemical properties of protein } \\
\text { tertiary structure }\end{array}$ & physico-protein & 45,730 & 9 \\
\hline $\begin{array}{l}\text { Relative location of } \mathrm{CT} \text { slices on axial } \\
\text { axis }\end{array}$ & CT-slices & 53,500 & $385 / 355$ \\
\hline Servo & servo & 167 & $4 / 15$ \\
\hline SML2010 & SML2010 & 4,137 & $20 / 18$ \\
\hline Stock portfolio & $\begin{array}{l}\text { stock-abs, stock-annual, stock-excess } \\
\text { stock-rel, stock-systematic, stock-total }\end{array}$ & 252 & 6 \\
\hline \multirow{2}{*}{ Student performance } & student-mat & 395 & $32 / 77$ \\
\hline & student-por & 649 & $32 / 56$ \\
\hline UJIIndoorLoc & UJ-lat, UJ-long & 21,048 & $528 / 373$ \\
\hline Yacht hydrodynamics & yacht-hydro & 308 & 6 \\
\hline YearPredictionMSD & year-prediction & 2,000 & 90 \\
\hline
\end{tabular}

Table 2: Collection of 83 datasets from the UCI repository. Each column reports: original name in the UCI repository; datasets created from the original one; number of patterns (or observations) and inputs, before and after preprocessing. 


\begin{tabular}{|c|c|}
\hline Excluded dataset & Reason \\
\hline Amazon access samples & Huge number of inputs $(20,000)$ \\
\hline Breast cancer Wisconsin (Prognostic) & Too few recurrent patterns $(47)$ \\
\hline Cargo 2000 Freight Tracking and Tracing & Less than 10 different output values (3) \\
\hline Challenger USA space shuttle O-ring & Too few patterns (23) and inputs (3) \\
\hline $\begin{array}{l}\text { Condition based maintenance of naval propul- } \\
\text { sion plants (compress output) }\end{array}$ & Less than 10 different output values (9) \\
\hline Container crane controller & Too few patterns (15) \\
\hline DrivFace & $\begin{array}{l}\text { Less than } 10 \text { different output values (4 sub- } \\
\text { jects) }\end{array}$ \\
\hline $\begin{array}{l}\text { Early biomarkers of Parkinsons disease based } \\
\text { on natural connected speech }\end{array}$ & Data are not available \\
\hline Educational process mining & Inputs and output for regression are not clear \\
\hline ElectricityLoadDiagrams & Huge number of inputs $(140,256)$ \\
\hline Fertility & Less than 10 different output values (2) \\
\hline $\begin{array}{l}\text { Gas sensor array drift dataset at different con- } \\
\text { centrations }\end{array}$ & Less than 10 different output values (7) \\
\hline $\begin{array}{l}\text { Gas sensor array exposed to turbulent gas mix- } \\
\text { tures }\end{array}$ & Huge number of inputs $(150,000)$ \\
\hline Gas sensor array under flow modulation & Less than 10 different output values (4) \\
\hline Geo-Magnetic field and WLAN & Data format very complex \\
\hline $\begin{array}{l}\text { Improved spiral test using digitized graphics } \\
\text { tablet for monitoring parkinsons disease }\end{array}$ & Data are not available \\
\hline Insurance Company Benchmark (COIL 2000) & Less than 10 different output values (3) \\
\hline KDC-4007 dataset Collection & Less than 10 different output values (8) \\
\hline KDD cup 1998 & Format too complex \\
\hline Las Vegas Strip & Less than 10 different output values (5) \\
\hline $\begin{array}{l}\text { News popularity in multiple social media plat- } \\
\text { forms }\end{array}$ & Data are text instead of numbers \\
\hline Noisy office & Format too complex (PNG images) \\
\hline Open university learning analytics & Format too complex \\
\hline Paper Reviews & Less than 10 different output values (5) \\
\hline $\begin{array}{l}\text { Parkinson disease spiral drawings using digi- } \\
\text { tized graphics tablet }\end{array}$ & Less than 10 different output values (3) \\
\hline Skillcraft1 master table & Less than 10 different output values (7) \\
\hline Solar flare & Less than 10 different output values \\
\hline Tamilnadu electricity board hourly readings & Less than 10 different output values (2) \\
\hline Tennis major tournament match statistics & Format problems \\
\hline Twin gas sensor arrays & Less than 10 different output values (4) \\
\hline UJIIndoorLoc-Mag & Output almost constant, format very complex \\
\hline wiki4HE & Less than 10 different output values (7) \\
\hline Wine quality (white/red) & Less than 10 different output values $(7 / 6)$ \\
\hline
\end{tabular}

Table 1: List of the UCI regression datasets which were excluded from this study with the reason to be discarded. In datasets with discrete outputs the number of different output (or response) values is between parentheses. 


\subsection{Datasets}

In the current research, we selected 48 of the 82 datasets ( 81 because the Air Quality dataset is repeated) listed as regression problems 4 by the UCI Machine Learning Repository [3]. The remaining 33 datasets were discarded due to the reasons listed in Table 1. The reason which leaded to discard a larger amount (17) of datasets was the reduced number of output (usually called response in Statistics) values, because the majority of the regression models are designed for datasets with continuous outputs and many different values, where an ordering relation has sense. Therefore, we excluded 17 datasets whose outputs have few values (specifically, less than 10), because including them in the dataset collection might favor some regression models with respect to others, thus biasing the results of the study. These datasets should be considered as ordinal classification instead of pure regression problems. Table 2 reports the collection of 83 datasets which we use in the current work, with their numbers of patterns (usually named observations in Statistics) and inputs (also called features or attributes). Some of the 48 original UCI regression datasets selected for this work generated several regression problems, one for each data column which can be used as output for regression. Thus, some UCI datasets (whose original names are listed in the column 1 of the tables) give several datasets in column 2 (e.g., the Air quality dataset gives five datasets named by us air-quality-CO, air-quality-NMHC, etc.). There are also discrepancies between data in Table 2 with respect to the documentation of the UCI ML repository, which are described in detail in Appendix A.

\footnotetext{
${ }^{4}$ http://archive.ics.uci.edu/ml/datasets.html?task=reg (visited February 5, 2018).
} 


\begin{tabular}{|c|c|}
\hline Dataset and details & Dataset and details \\
\hline 3Droad: 4: altitude & geo-long: 118: longitude; same file \\
\hline airfoil : 6: scaled sound pressure & geo-music-lat : 69: latitude; default file \\
\hline air-quality-CO : 3: PT08.S1; 1,2,7,9,11,12 & geo-music-long : 70: longitude; same file \\
\hline air-quality-NMHC : 7 : PT08.S2; 1,2,4,9,11,12 & gps-trajectory* $^{*}$ : speed; $1,9,12,13$; tracks file \\
\hline air-quality-NO2 : 10: PT08.S4; 1,2,4,7,9,12 & greenhouse-net: 16: synthetic; pasted all files \\
\hline air-quality-NOx : 9: PT08.S3; $1,2,4,7,11,12$ & household-consume* : 3: global_active_power \\
\hline air-quality-O3 : 11: PT08.S5; 1,2,4,7,9,11 & housing: 14: MEDV \\
\hline appliances-energy : 2: appliances; 1 & KEGG-reaction* $^{*}: 29$ : edgeCount; 1 \\
\hline auto-MPG ${ }^{*}: 1: \mathrm{mpg}$ & KEGG-relation : 24: ClusteringCoefficient; 1 \\
\hline automobile : 26: price : & online-news : 60: shares \\
\hline bike-day : 16: cnf; 1,$2 ;$ day.csv & $\begin{array}{l}\text { park-motor-UPDRS : 5: motor_UPDRS; } 12,3,4 \text {, } \\
6\end{array}$ \\
\hline bike-hour : 17: cnf; 1,2; hour.csv & park-speech : 28: UPDRS; train_data.txt \\
\hline blog-feedback : 281: target; pasted all files & park-total-UPDRS : $6:$ total_UPDRS; $1,2,3,4,5$ \\
\hline buzz-twitter : 78: discussions : Twitter.data & physico-protein : 1: RMSD \\
\hline combined-cycle : 5: PE; Folds5x2_pp.csv & servo : $5:$ class \\
\hline com-crime* : 128: ViolentCrimesPerPop;1-5 & slump : 8: slump \\
\hline $\begin{array}{l}\text { com-crime-unnorm* }{ }^{*}: 146: \text { ViolentCrimesPerPop; } \\
1-5,130-145,147\end{array}$ & slump-comp : 10: comp. strength \\
\hline com-hd : 10: ERP; 1,2 & slump-flow : 9: flow \\
\hline compress-stren : 9: ccs; Concrete_data.xls & $\begin{array}{l}\text { SML2010* : } 3: \text { dining-room temperature; 1,2,4; } \\
\text { both files }\end{array}$ \\
\hline cond-turbine : 18: GT Turbine; 17; data.txt & stock-exchange : 10: EM; 1 \\
\hline CT-slices : 386: reference & student-mat : 33: G3; G1, G2 \\
\hline cuff-less $^{*}: 2:$ ABP & student-por : 33: G3; G1, G2 \\
\hline energy-cool : 10: cool & UJ-lat : 522: latitude; both files \\
\hline energy-heat : 9: heat & UJ-long : 521: longitude; both files \\
\hline \multicolumn{2}{|l|}{ facebook-comment : 54; Features_Variant_1.csv } \\
\hline \multicolumn{2}{|l|}{ facebook-metrics : 1} \\
\hline forestfires : 13: area & $\begin{array}{lllll}\text { video-transcode } \quad: & 21: & \text { utime; } & \text { transcod- } \\
\text { ing_mesurment.tsv } & & & \\
\end{array}$ \\
\hline gas-dynamic-CO : 2: CO conc; 1 & yacht-hydro : 7: resistance \\
\hline gas-dynamic-methane : 2: Methane; 1 & year-prediction : 1: year \\
\hline geo-lat : 117: latitude; chromatic file & \\
\hline
\end{tabular}

Table 3: Information about datasets used in the current work: column number and name (if exists) used as output; removed columns (e.g., time marks or other outputs) where corresponds; files used, in datasets where several files are available; *: means that dataset contains missing patterns, which we replaced by the column mean.

Although several datasets in Table 2 contain several ten thousand patterns, and even half (buzz-twitter), one (greenhouse-net) and two million patterns (household-consume), the current study is not oriented to large-scale datasets because the available implementations of the majority of regression models would not work on such large datasets due to memory errors or exces- 
sive time. Thus, including large-scale datasets on the current study would bias the results and conclusions, limiting the comparison to those models with implementations that could be run on large data and favoring them over the remaining ones. Such a study for large-scale datasets would require a separate and completely different work which falls outside the scope of the current paper. Even discarding large-scale datasets, some models in our study are not able to train and test with some large datasets of our collection due to the limited RAM memory, although we set a maximum size of 128 GB. Besides, some other models spend a long time to finish, so we fixed a maximum run-time of 48 hours and labeled any model that could not finish within this time lapse as failing for this dataset. As usual, the output was pre-processed using the Box-Cox transformation [4] in order to make it more similar to a symmetric uni-modal distribution, with the boxcox function (MASS package) of the $\mathrm{R}$ statistical computing language [5]. In the greenhouse-net and com-crime-unnorm datasets, the decimal logarithm of the inputs are used, due to the wide range of many inputs. The constant, repeated and collinear input:5 are removed from all the datasets. Specifically, the $\mathrm{lm}$ function in the stats $\mathrm{R}$ package is used to calculate the coefficients of the linear model trained on the whole dataset, and the inputs with NA (not available) coefficients in the linear model are removed. This reason leads e.g. the Blog feedback dataset to reduce its inputs from 280 to 13 . The rationale behind this is that constant, repeated or collinear inputs lead many models to develop calculations with singular matrices, so it is useful to remove these

\footnotetext{
${ }^{5} \mathrm{An}$ input is considered collinear when it can be calculated as a linear combination of other inputs.
} 
inputs in order to avoid the subsequent errors. On the other hand, the inputs with discrete values are replaced by dummy (also named indicator) inputs. For each discrete input (often named nominal variables in Statistics) with $n$ values, it is replaced by $n-1$ dummy binary inputs. The first value of the original discrete input is codified as zero values for the $n-1$ dummy inputs; the second value is codified as 1 in the first dummy variable and zero in the remaining ones, and so on. Therefore, those datasets with discrete inputs increase the number of inputs, so that e.g. the student-mat dataset (Table 2, second column) increases its inputs from 32 to 77 due to the presence of discrete inputs. In Table 2 the datasets whose "\#inputs" column shows two numbers (i.e. 8/23), the first is the number of inputs of the original UCI dataset, and the second is the number of inputs used effectively in our experiments, after removing those inputs which are constant, repeated or collinear, and after replacing discrete inputs by their corresponding dummy variables. Those datasets with only one number in the \#inputs column means that no input was removed nor added. Table 3 reports the name and number of the attribute used as output for each dataset. It also specifies the numbers of the columns that were discarded (if any), due to being useless (e.g., times, dates, names, etc.) or because they are alternative outputs (in datasets with several outputs to be predicted) which can not be used as inputs (e.g., latitude can not be used as input for UJ-long dataset in Table 21). In those datasets with more than one file, the table specifies the files used. An asterisk $\left(^{*}\right)$ identifies datasets with missing values, which are replaced by the mean of the non-missing values of that column. Note that applying further sophisticated management techniques to inputs with missing values might allow to extract 
some information out of them in order to raise the prediction accuracy.

\subsection{Regression models}

We apply a wide collection of 77 models which belong to several families. All the files (data, programs and results) are publicly available6. The majority of them (74 models) are selected from the list of model 7 included in the Classification and Regression Training (caret) R package [6]. We discarded 52 caret models listed in Table 4, either because they are equivalent to other models already included in our study (which are listed in the "Equivalence" columns of the upper part of the table), due to run-time errors or because they can not be used for regression (listed in the lower part of the table). Instead of using the train function of the caret package, we ran the models directly using the corresponding $\mathrm{R}$ packages (see the detailed list of models below), in order to control the execution of each single model. Besides, the direct execution allows us to use the same configuration (e.g., the same training and test patterns) as other four popular models, implemented in other platforms, that we included in our study although they do not belong to the caret model list (see the link in the above footnote). These models are the deep learning neural network (named dlkeras in our study), using the module Keras, configured for Theano [7], in the Python programming language [8]; the $\varepsilon$-support vector regression (named svr), implemented by the LibSVM library [9] and accessed via the $\mathrm{C}++$ interface; the generalized regression neural network and extreme learning machine with Gaussian kernel

${ }^{6}$ https://nextcloud.citius.usc.es/index.php/s/Yb8LZQQFrgckjFk (visited December 14, 2018).

[http://topepo.github.io/caret/available-models.html (visited April 27, 2017). 


\begin{tabular}{|c|c|c|c|}
\hline Family & Regression models & Family & Regression models \\
\hline \multirow{2}{*}{ Linear regression $(\mathrm{LR})$} & 1. $\operatorname{lm}[11]$ & \multirow{3}{*}{$\begin{array}{l}\text { Least absolute shrinkage } \\
\text { and selection operator } \\
\text { (LASSO) }\end{array}$} & 21. lasso [12 \\
\hline & 2. $\operatorname{rlm}[13]$ & & 22. relaxo [14] \\
\hline \multirow{4}{*}{$\begin{array}{l}\text { Generalized linear } \\
\text { regression (PLM) }\end{array}$} & 3. penalized [15] & & 23. lars [16] \\
\hline & 4. enet [12] & \multirow{3}{*}{ Ridge } & 24. ridge $[12]$ \\
\hline & 5. glmnet [17] & & 25. spikeslab [18] \\
\hline & 6. glmSAIC [19] & & 26. foba $[20]$ \\
\hline \multirow{2}{*}{ Additive models (AM) } & 7. gam [21] & \multirow{3}{*}{ Bayesian models (BYM) } & 27. bayesglm [22] \\
\hline & 8. earth [23] & & 28. brnn [24] \\
\hline \multirow{2}{*}{ Least squares (LS) } & 9. nnls [25] & & 29. bMachine [26] \\
\hline & 10. krlsRadial [27] & \multirow{3}{*}{ Gaussian processes (SGP) } & 30. gprLin [28] \\
\hline \multirow{11}{*}{$\begin{array}{l}\text { Projection methods } \\
\text { (PRJ) }\end{array}$} & 11. spls [29] & & 31. gprRad 28] \\
\hline & 12. simpls 30] & & 32. gprPol [28] \\
\hline & 13. kpls [31] & \multirow{3}{*}{ Quantile regression (QTR) } & 33. rqlasso 32 \\
\hline & 14. wkpls [33] & & 34. rqne 34 \\
\hline & 15. enpls.fs [35] & & 35. qrnn [36] \\
\hline & 16. plsRglm [37] & Nearest neighbors (NN) & 36. kknn [38] \\
\hline & 17. ppr [39] & \multirow{5}{*}{ Regression trees (RGT) } & 37. rpart [40] \\
\hline & 18. pcr [41] & & 38. nodeHarvest [4] \\
\hline & 19. icr [43] & & 39. ctree2 [44] \\
\hline & \multirow{2}{*}{ 20. superpc [45] } & & 40. partDSA [46] \\
\hline & & & 41. evtree [47] \\
\hline
\end{tabular}

Table 5: List of regression models and references grouped by families (see Appendix B for a brief description of each model).

these cases, although the caret model list specifies that hyperparameter as tunable, in the practice only one value is used. The list of hyperparameter values which are used in our experiments for a model and dataset is included in the file results_model_dataset.dat, where model and dataset stand for the names of the regression model and dataset, respectively, which is placed in the directory results/dataset/model_implem, where implem may be $R$, C, Python or Matlab. For some models (ridge, rlm, mlpWD, mlpWDml, dnn, krlsRad and icr), the value list provided by the getModelInfo function was not valid due to several reasons, so we directly specify the hyperparameter values used for tuning in the file programs/R/initialize.R. The models in Matlab, $\mathrm{C}++$ and Python use pre-specified values, listed in the script 


\begin{tabular}{|c|c|c|c|}
\hline Family & Regression models & Family & Regression models \\
\hline \multirow{3}{*}{$\begin{array}{l}\text { Regression rules } \\
\text { (RGR) }\end{array}$} & 42. M5 [48] & \multirow{4}{*}{$\begin{array}{l}\text { Boosting (BST) } \\
\text { (continued) }\end{array}$} & 60. gbm [49] \\
\hline & 43. cubist [50] & & 61. blackboost 51 \\
\hline & 44. SBC [52] & & 62. xgbTree [53] \\
\hline \multirow{6}{*}{ Random forests (RF) } & 45. $\operatorname{rf}[54]$ & & 63. xgbLinear [53] \\
\hline & 46. Boruta [55] & \multirow{9}{*}{$\begin{array}{l}\text { Neural networks } \\
(\mathrm{NET})\end{array}$} & 64. mlWD [56] \\
\hline & 47. RRF [57] & & 65. mlWDml [56] \\
\hline & 48. cforest [58] & & 66. avNNet [6] \\
\hline & 49. qrf [59] & & 67. $\operatorname{rbf}[56]$ \\
\hline & 50. extraTrees [60] & & 68. grnn 61 \\
\hline \multirow{3}{*}{ Bagging (BAG) } & 51. bag 62 & & 69. elm [63] \\
\hline & 52. bagEarth [6] & & 70. kelm [63] \\
\hline & 53. treebag [64] & & 71. pcaNNet [6] \\
\hline \multirow{6}{*}{ Boosting (BST) } & 54. rndGLM [65] & & 72. bdk [66] \\
\hline & 55. BstLm [53] & \multirow{2}{*}{ Deep learning (DL) } & 73. dlkeras 67 \\
\hline & 56. bstSm [53] & & 74. dnn [68] \\
\hline & 57. bstTree [53] & \multirow{3}{*}{$\begin{array}{l}\text { Support vector } \\
\text { regression (SVR) }\end{array}$} & 75. svr [9] \\
\hline & 58. glmboost [69] & & 76. svmRad [70] \\
\hline & 59. gamboost [69] & & 77. rvmRad [71] \\
\hline
\end{tabular}

Table 6: Continuation of Table 5 .

run_model_dataset.sh, which are the same for all datasets. Tables 5 and 6] list the the collection of 77 regression models used in this work, grouped by families, which are described in Appendix B, specifying the software implementation ( $\mathrm{R}$ package or other platforms), their tunable hyperparameters and the values used.

\section{Results and discussion}

The experimental work [72] uses the following methodology: for each dataset with less than 10,000 patterns, $N=500$ random partitions are generated, using the $50 \%$ of the patterns for training, $25 \%$ for validation (in hyperparameter tuning) and $25 \%$ for test. For each dataset with more than 10,000 patterns, a 10-fold cross validation is developed, so there are $N=10$ training, validation and test percentages. The rationale is to limit the compu- 
tational overhead of 500 trials to smaller datasets, using a lighter methodology (10-fold), although statistically less significant, for larger datasets. Each regression model is trained on the training partitions for each combination of its hyperparameter values, and it is tested on its corresponding validation partition. The performance measures used are the root mean square error (RMSE), the squared correlation $\left(R^{2}\right)$ and the mean absolute error (MAE). We use these three different measures in order to give more significance to the results, which in this way can be observed from three different points of view, and also in order to evaluate whether they are coherent suggesting similar conclusions. For each combination of hyperparameter values, the average RMSE over the validation partitions is calculated, and the combination with the lowest average RMSE is selected for testing (quantile regression models as rqlasso, rqnc and qrnn are designed to optimize the quantile error, which is used instead of RMSE). Finally, the model is trained on the training partitions using the selected combination of its hyperparameter value and tested on the test partitions. The performance measurements are the RMSE, $R^{2}$ and MAE between the true and predicted output values concatenated for the $N$ test sets. Note that the $R^{2}$ is calculated using the predicted and true outputs for the test patterns, while it is often used to measure the percentage of variance explained by the model on the training patterns. Those regression models which lack tunable hyperparameters are trained on the training partitions and tested on the corresponding test partitions, and the average RMSE, $R^{2}$ and MAE over the test partitions are the quality measurements. Some models which are specially sensitive to collinear inputs are trained, for each partition, using only those inputs which are not collinear. Although collinear 
inputs have been removed from the dataset in the initial preprocessing, for certain partitions some inputs in the training set may be collinear despite of being not collinear considering the whole dataset. To avoid the subsequent errors, these inputs are discarded for these models. All the continuous inputs and the output are standardized to have zero mean and standard deviation one, using the mean and deviation calculated in each training partition.

We run this collection of 77 regression models over 83 datasets, developing a total of 6,391 experiments, which were developed on a cluster whose nodes are equipped with 64 Intel Xeon E5-2650L processors and 4 GB of RAM memory each processor, although those regression models which required more memory with large data sets were run using several processors and up to 128 GB of RAM memory. Since certain models failed for some datasets, we developed a preliminar study to evaluate the datasets according to their size, given by its population, and "difficulty", estimated by the $R^{2}$ achieved by the linear regression model $(\mathrm{lm})$. We selected $1 \mathrm{~m}$ because it is a classical approach which can be considered as a baseline reference for other models and it does not require large time nor memory, so it does not fail in any dataset. Figure 1 plots $R_{l m}^{2}$ for all the datasets vs. their populations $N_{p}$. According to this plot, we divided the datasets into four groups: group SD includes 20 datasets with $N_{p}<5,000$ and $R_{l m}^{2}<0.6$, i.e., small-difficult datasets; group SE includes 23 datasets with $R_{l m}^{2} \geq 0.6$ and $N_{p}<5,000$ (small-easy datasets); group LD with 33 datasets where $R_{l m}^{2}<0.6$ and $N_{p} \geq 5,000$ (large-difficult datasets); and group LE with 7 datasets where $R_{l m}^{2} \geq 0.6$ and $N_{p} \geq 5,000$ (large-easy datasets). Table 7 lists the datasets of each group.

In order to compare the $R^{2}$ values achieved by the regression models over 


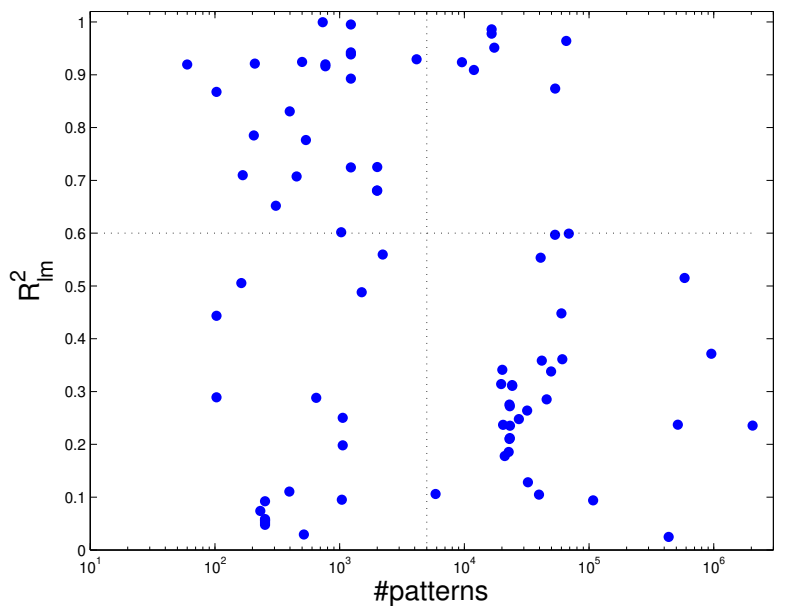

Figure 1: Values of $R^{2}$ achieved by $\mathrm{lm}$ for all the datasets plotted against their populations (in logarithmic scale), dividing datasets in groups small-difficult (SD, lower left quarter of the figure), small-easy (SE, upper left), large-difficult (LD, lower right) and large-easy (LE, upper right).

all the datasets, averaging would weight more those datasets with high $R^{2}$, favoring models which perform well in easy datasets and biasing the results. In order to do a neutral comparison, the solution is to average over all the datasets the model positions in a ranking sorted by decreasing $R^{2}$, instead of directly averaging $R^{2}$ values, because the model positions belong to the same range for all the datasets. This is done using the Friedman ranking [73] of the $R^{2}$ coefficient, which evaluates the position where each regression model is placed, in average over all the datasets, when $R^{2}$ is sorted by decreasing values. The $R^{2}$ Friedman ranking of the $M=77$ models over the $D=83$ datasets can be calculated as follows. For each dataset $d=1, \ldots, D$, the $R^{2}$ values of all the models are sorted decreasingly. For each model $m=1, \ldots, M$ let $p_{m d}$ be its position in dataset $d$. The Friedman rank $F_{m}$ of model $m$ is defined as $F_{m}=\frac{1}{D} \sum_{d=1}^{D} p_{m d}$, i.e., the average position of model $m$ over all 
the sortings of $R^{2}$ for the different datasets. For example, a model with rank 5 achieves the 5 th highest $R^{2}$ coefficient in average over all the datasets.

A number of run-time errors happened for certain models and datasets. There are more errors in large datasets, because some model implementations may not be designed to process large amounts of data. When a model fails for a dataset (because it overcomes the maximum allowed time of 48 hours, because it requires more than $128 \mathrm{~GB}$ of RAM, or due to other reasons), and in order to calculate the Friedman ranking, its $R^{2}$ is intended to be zero, while its RMSE and MAE are assigned as:

$$
\begin{array}{r}
R M S E=\max \left\{\max _{m \in \mathcal{R}}\left[R M S E_{m}\right], \sqrt{\frac{1}{N} \sum_{i=1}^{N}\left(t_{i}-\bar{t}_{i}\right)^{2}}\right\} \\
M A E=\max \left\{\max _{m \in \mathcal{R}}\left[M A E_{m}\right], \frac{1}{N} \sum_{i=1}^{N}\left|t_{i}-\bar{t}\right|\right\}
\end{array}
$$

where $\mathcal{R}$ is the set of models which did not fail in that dataset, $t_{i}$ is the true output for test pattern $i$ and $N$ is the number of test patterns. Besides, denoting by $k$ the test partition to which pattern $i$ belongs, $\bar{t}_{i}$ is the mean of the true output values over the patterns in the $k$-th training partition. The rationale behind this is that a regression model which fails behaves as if it would predict the mean of the true output values for all the test patterns, so it should be the last of the list. For some models, the errors happen only during tuning for some partitions, which are not considered to calculate the average RMSE corresponding to that combination of hyper-parameter values. When a model fails for a given combination of hyper-parameter values and all the 
partitions, that combination is not selected for testing. When a model fails for all the combinations of hyper-parameter values, or when it fails for some test partition, the model is considered that fails for that dataset. Overall, the number of experiments where the model failed is 1,205 and represents $18.85 \%$ of the 6,391 experiments.

\begin{tabular}{|c|c|}
\hline Group (\#datasets) & Datasets \\
\hline SD (20): small-difficult & $\begin{array}{l}\text { airfoil com-crime-unnorm csm1415 forestfires geo-lat geo-long geo-music- } \\
\text { lat geo-music-long gps-trajectory park-speech slump slump-flow stock-abs } \\
\text { stock-annual stock-excess stock-rel stock-systematic stock-total student-mat } \\
\text { student-por }\end{array}$ \\
\hline SE (23): small-easy & $\begin{array}{l}\text { air-quality-CO air-quality-NMHC air-quality-NO2 air-quality-NOx air- } \\
\text { quality-O3 automobile auto-mpg bike-day com-crime com-hd compress-stren } \\
\text { daily-demand energy-cool energy-heat facebook-metrics gas-dynamic-CO gas- } \\
\text { dynamic-methane housing servo slump-comp SML2010 stock-exchange yacht- } \\
\text { hydro }\end{array}$ \\
\hline$\overline{L D}$ (33): large-difficult & $\begin{array}{l}\text { 3Droad appliances-energy beijing-pm25 blog-feedback buzz-twitter cuff-less } \\
\text { dynamic-features facebook-comment greenhouse-net household-consume } \\
\text { KEGG-relation online-news park-motor-UPDRS park-total-UPDRS } \\
\text { physico-protein pm25-beijing-dongsi pm25-beijing-dongsihuan pm25- } \\
\text { beijing-nongzhanguan pm25-beijing-us-post pm25-chengdu-caotangsi pm25- } \\
\text { chengdu-shahepu pm25-chengdu-us-post pm25-guangzhou-5th-middle-school } \\
\text { pm25-guangzhou-city-station pm25-guangzhou-us-post pm25-shanghai- } \\
\text { jingan pm25-shanghai-us-post pm25-shanghai-xuhui pm25-shenyang- } \\
\text { taiyuanji pm25-shenyang-us-post pm25-shenyang-xiaoheyan video-transcode } \\
\text { year-prediction }\end{array}$ \\
\hline LE (7): large-easy & $\begin{array}{l}\text { bike-hour combined-cycle cond-turbine CT-slices KEGG-reaction UJ-lat UJ- } \\
\text { long }\end{array}$ \\
\hline
\end{tabular}

Table 7: Groups of datasets according to its size (small/large) and complexity (easy/difficult).

\subsection{Discussion by dataset group}

Table 8 reports the 20 best regression models according to the Friedman ranking of $R^{2}$, RMSE and MAE for the datasets of group SD, which includes 20 small-difficult datasets. For each model in the $R^{2}$ ranking, the percentage of datasets where it failed is also reported (column \%Error). The last two columns report the models which achieved the best $R^{2}$ for some dataset and the percentage of datasets where this happened. First of all, 
penalized achieves the first positions in the three rankings, being the best $R^{2}$ for $25 \%$ of datasets. ExtraTrees, rf and kelm are the following in the $R^{2}$ ranking, although the former achieves much lower positions in RMSE and MAE rankings. Specifically, extraTrees achieves the best $R^{2}$ in $40 \%$ of the datasets, although it fails in $5 \%$ of them, so it can be considered less regular as penalized. Other good positions in the $R^{2}$ ranking are for qrf and bstTree, followed by avNNet, svr and svmRad, Gaussian process (gprRad and gprPol), bagEarth and cubist, which achieves the best $R^{2}$ for $10 \%$ of datasets.

\begin{tabular}{|c|c|c|c|c|c|c|c|c|c|}
\hline & \multicolumn{3}{|c|}{$R^{2}$} & \multicolumn{2}{|c|}{ RMSE } & \multicolumn{2}{|c|}{ MAE } & \multicolumn{2}{|c|}{ Best $R^{2}$} \\
\hline Pos. & Model & Rank & $\%$ Error & Model & Rank & Model & Rank & Model & \%Best \\
\hline 1 & penalized & 8.45 & 0.0 & penalized & 9.65 & penalized & 13.40 & extraTrees & 40.0 \\
\hline 2 & extraTrees & 13.05 & 5.0 & kelm & 13.15 & svmRad & 13.90 & penalized & 25.0 \\
\hline 3 & $\mathrm{rf}$ & 15.35 & 5.0 & gprPol & 14.45 & svr & 15.70 & cubist & 10.0 \\
\hline 4 & kelm & 19.15 & 5.0 & bagEarth & 17.55 & kelm & 16.25 & brnn & 10.0 \\
\hline 5 & qrf & 20.75 & 0.0 & svmRad & 18.00 & bstTree & 19.15 & rbf & 5.0 \\
\hline 6 & bstTree & 21.00 & 0.0 & cforest & 18.65 & gprPol & 19.25 & qrf & 5.0 \\
\hline 7 & avNNet & 21.25 & 5.0 & bstTree & 19.10 & cubist & 19.65 & bagEarth & 5.0 \\
\hline 8 & svr & 22.20 & 10.0 & svr & 19.35 & bagEarth & 19.75 & - & - \\
\hline 9 & svmRad & 23.15 & 5.0 & enet & 21.50 & cforest & 21.45 & - & - \\
\hline 10 & gprRad & 23.20 & 0.0 & BstLm & 22.75 & qrf & 23.35 & - & - \\
\hline 11 & RRF & 24.10 & 20.0 & glmboost & 23.80 & avNNet & 23.85 & - & - \\
\hline 12 & bagEarth & 24.10 & 0.0 & gbm & 24.20 & gbm & 24.35 & - & - \\
\hline 13 & gprPol & 24.35 & 0.0 & foba & 24.70 & grnn & 27.00 & - & - \\
\hline 14 & gbm & 24.60 & 0.0 & bMachine & 25.30 & gprRad & 28.35 & - & - \\
\hline 15 & cubist & 26.20 & 5.0 & grnn & 26.25 & extraTrees & 29.05 & - & - \\
\hline 16 & ridge & 27.85 & 0.0 & spls & 27.25 & $\mathrm{rf}$ & 29.15 & - & - \\
\hline 17 & treebag & 29.45 & 0.0 & spikeslab & 27.25 & BstLm & 29.45 & - & - \\
\hline 18 & foba & 29.55 & 0.0 & $\mathrm{rf}$ & 27.90 & treebag & 29.50 & - & - \\
\hline 19 & spls & 29.85 & 0.0 & lars & 28.35 & rqlasso & 29.75 & - & - \\
\hline 20 & lars & 30.25 & 0.0 & avNNet & 28.90 & glmboost & 29.95 & - & - \\
\hline
\end{tabular}

Table 8: List of the 20 best regression models according to the Friedman rank of $R^{2}$, RMSE and MAE for dataset group SD, with 20 small-difficult datasets. The last two columns list the models which achieve the best $R^{2}$ for some dataset, sorted by decreasing number of datasets.

Since this group includes only small datasets, most models exhibit low error percentages (i.e., most models never or rarely fail on datasets of this 
group), although some models with errors achieve good positions, e.g. svr (10\% of errors), RRF (20\%), extraTrees and cubist (5\% each one). Besides, qrnn and nodeHarvest are very slow and they were shutdown after $48 \mathrm{~h}$. for the 20 datasets of this group. Considering memory errors, rndGLM is the model which requires more memory, overcoming the memory and time limits in 1 and 5 datasets of this group, respectively.

\begin{tabular}{|c||c|c|c||c|c||c|c||c|c|}
\hline & \multicolumn{3}{|c||}{$R^{2}$} & \multicolumn{2}{c|}{ RMSE } & \multicolumn{2}{c||}{ MAE } & \multicolumn{2}{c|}{ Best $R^{2}$} \\
\hline Pos. & Model & Rank & \%Error & Model & Rank & Model & Rank & Model & $\%$ Best \\
\hline \hline 1 & cubist & 6.48 & 0.0 & cubist & 6.26 & cubist & 5.65 & cubist & 21.7 \\
2 & avNNet & 10.13 & 4.3 & avNNet & 10.04 & avNNet & 10.96 & avNNet & 13.0 \\
3 & bstTree & 12.57 & 0.0 & bstTree & 12.39 & bstTree & 13.70 & extraTrees & 13.0 \\
4 & gbm & 12.87 & 0.0 & gbm & 12.74 & ppr & 13.91 & gbm & 8.7 \\
5 & bagEarth & 14.57 & 0.0 & bagEarth & 14.30 & gbm & 13.96 & penalized & 8.7 \\
6 & ppr & 14.65 & 0.0 & ppr & 14.52 & bagEarth & 15.96 & bMachine & 8.7 \\
7 & bMachine & 14.96 & 4.3 & bMachine & 15.22 & bMachine & 16.70 & kelm & 4.3 \\
8 & extraTrees & 17.13 & 8.7 & earth & 18.35 & M5 & 16.83 & M5 & 4.3 \\
9 & earth & 18.57 & 0.0 & kelm & 18.65 & qrf & 17.43 & rf & 4.3 \\
10 & kelm & 18.70 & 17.4 & extraTrees & 18.87 & extraTrees & 18.00 & brnn & 4.3 \\
11 & rf & 19.26 & 4.3 & rf & 19.65 & kelm & 18.74 & bagEarth & 4.3 \\
12 & M5 & 20.30 & 0.0 & M5 & 19.87 & rf & 19.70 & bstTree & 4.3 \\
13 & RRF & 22.43 & 8.7 & RRF & 22.61 & earth & 21.00 & - & - \\
14 & qrf & 22.48 & 0.0 & qrf & 23.43 & brnn & 22.48 & - & - \\
15 & brnn & 23.65 & 21.7 & brnn & 23.61 & RRF & 22.83 & - \\
16 & gprPol & 24.00 & 4.3 & gprPol & 24.22 & pcaNNet & 24.17 & - & - \\
17 & pcaNNet & 25.04 & 0.0 & pcaNNet & 25.09 & gprPol & 25.78 & - & - \\
18 & dlkeras & 27.35 & 0.0 & dlkeras & 27.61 & rqlasso & 26.52 & - & - \\
19 & Boruta & 27.43 & 17.4 & Boruta & 27.78 & cforest & 27.13 & - & - \\
20 & enet & 28.52 & 0.0 & enet & 28.09 & Boruta & 27.70 & - & - \\
\hline
\end{tabular}

Table 9: List of the 20 best regression models according to the Friedman rank of $R^{2}$, RMSE and MAE over 23 datasets of group SE (small-easy).

Considering small-easy datasets (group SE, 23 datasets, table 9), the three rankings are even more coherent than for group SD, sharing the first three positions: cubist, which achieves the best $R^{2}$ for $21.7 \%$ of datasets, avNNet (the best $R^{2}$ for $13 \%$ of datasets) and bstTree. Penalized is not present in this list (although it is the best in $8.7 \%$ of datasets), but gbm and bMachine (which are the bests in $8.7 \%$ of datasets), bagEarth, ppr, 

RRF and qrf.

\begin{tabular}{|c||c|c|c||c|c||c|c||c|c|}
\hline \multicolumn{1}{|c||}{} & \multicolumn{3}{|c||}{$R^{2}$} & \multicolumn{2}{c|}{ RMSE } & \multicolumn{2}{c|}{ MAE } & \multicolumn{2}{c|}{ Best $R^{2}$} \\
\hline Pos. & Model & Rank & \%Error & Model & Rank & Model & Rank & Model & $\%$ Best \\
\hline 1 & M5 & 9.48 & 0.0 & M5 & 9.39 & M5 & 9.55 & extraTrees & 48.5 \\
2 & cubist & 12.39 & 15.2 & gbm & 12.61 & kknn & 12.55 & bstTree & 12.1 \\
3 & gbm & 12.48 & 3.0 & cubist & 12.70 & cubist & 12.61 & cubist & 9.1 \\
5 & xgbTree & 14.24 & 6.1 & xgbTree & 14.15 & gbm & 13.42 & dlkeras & 6.1 \\
6 & kknn & 14.48 & 12.1 & kknn & 14.48 & xgbTree & 14.82 & xgbTree & 6.1 \\
7 & bstTree & 15.12 & 12.1 & bstTree & 15.27 & bstTree & 16.00 & ppr & 3.0 \\
8 & blackboost & 16.79 & 0.0 & blackboost & 17.27 & grnn & 17.33 & kknn & 3.0 \\
9 & dlkeras & 18.36 & 15.2 & pcaNNet & 18.33 & blackboost & 17.70 & M5 & 3.0 \\
10 & svr & 18.58 & 27.3 & svr & 18.45 & svr & 18.76 & rf & 3.0 \\
\hline 11 & pcaNNet & 18.76 & 0.0 & dlkeras & 18.67 & pcaNNet & 18.82 & qrf & 3.0 \\
12 & grnn & 19.70 & 18.2 & ppr & 19.48 & dlkeras & 19.18 & bMachine & 3.0 \\
13 & ppr & 19.73 & 3.0 & grnn & 19.52 & ppr & 19.58 & - & - \\
14 & qrf & 21.33 & 27.3 & qrf & 21.27 & qrf & 20.30 & - \\
15 & svmRad & 21.88 & 24.2 & svmRad & 21.79 & svmRad & 20.58 & - \\
16 & earth & 22.52 & 0.0 & earth & 22.21 & extraTrees & 22.33 & - \\
17 & extraTrees & 23.03 & 27.3 & bag & 22.91 & bag & 22.61 & - \\
18 & bag & 23.03 & 15.2 & avNNet & 23.42 & earth & 22.88 & - \\
19 & bMachine & 24.76 & 24.2 & bMachine & 24.64 & bMachine & 25.24 & - \\
20 & cforest & 25.79 & 27.3 & cforest & 25.91 & rpart & 26.00 & - & - \\
\hline
\end{tabular}

Table 10: List of the 20 best regression models according to the Friedman rank of $R^{2}$, RMSE and MAE for the 33 datasets of group LD (large-difficult).

extraTrees (the best in $13 \%$ of datasets), earth and kelm are in positions 410. Other models with good results are rf, M5 (the best for $4.3 \%$ of datasets), RMSE and MAE for the 33 datasets of group LD (large-difficult).

In large-difficult datasets (group LD, 33 datasets, table 10), the M5 achieves the first positions in the three rankings (although it achieves the best $R^{2}$ only in $3 \%$ of datasets), followed by cubist (which achieves the best $R^{2}$ and errors in $9.1 \%$ and $15.2 \%$ of datasets, respectively) and gbm. Other models with good performance are xgbTree, knn, bstTree, blackboost, dlkeras (15.2\% of errors), svr (with errors in $27.3 \%$ of datasets) and pcanNet. The high error frequency of several models (either by overcoming limits on memory or time) is due to the large size of datasets in this group. ExtraTrees also overcomes the maximum time in $27.3 \%$ of datasets and, as in groups SD and 
$\mathrm{SE}$, it achieves the best $R^{2}$ for more datasets (48.5\%). Specifically, it achieves the highest $R^{2}$ for 13 of the 16 datasets created from the original PM2.5 Data 5 Chinese Cities dataset in the UCI repository. In these datasets svr and kelm were run with a lower number of hyperparameter values $(\{0.125,0.5,1$, $4,16\}$ and $\{0.003910 .015620 .12514\}$ for $C$ and $\gamma$, respectively), in order to avoid overcoming the maximum run time. The models wkpls, gprPol, krlsRad, rvmRad, SBC and qrnn failed for the 33 datasets of this group.

\begin{tabular}{|c|c|c||c|c|c|c|c|c|c|c|c|}
\hline Pos. & Model & Rank & Pos. & Model & Rank & Pos. & Model & Rank & Pos. & Model & Rank \\
\hline \hline 1 & M5 & 8.1 & 6 & pcaNNet & 15.4 & 11 & rpart & 23.5 & 16 & avNNet & 26.6 \\
\hline 2 & gbm & 9.8 & 7 & earth & 18.6 & 12 & treebag & 24.1 & 17 & svmRad & 26.8 \\
\hline 3 & blackboost & 10.9 & 8 & kknn & 19.4 & 13 & ctree2 & 25.1 & 18 & enet & 26.8 \\
\hline 4 & xgbTree & 14.6 & 9 & bstTree & 19.8 & 14 & elm & 26.2 & 19 & bag & 26.9 \\
\hline 5 & ppr & 14.8 & 10 & cubist & 20.4 & 15 & svr & 26.2 & 20 & dlkeras & 27.9 \\
\hline
\end{tabular}

Table 11: Friedman rank of $R^{2}$ (first 20 models) of group LD (large-difficult datasets) discarding PM2.5 Data Chinese Cities datasets.

The PM2.5 Data 5 Chinese Cities datasets represent almost the half of the 33 datasets in this group. Since this fact might bias the results, we calculated the $R^{2}$ Friedman rank discarding these 16 datasets (Table 11). In this case, the best model is M5 again, cubist descends to the 10th position, replaced by gbm and followed by blackboost, xgbTree and ppr, while extraTrees leaves the top-20.

The rankings of group LE (large-easy, Table 12) is very similar to group LD: the M5 achieves again the best position in the rankings of $R^{2}$, RMSE and MAE, followed by the same models as the previous group: cubist (which achieves the best $R^{2}$ in $42.9 \%$, and errors in $14.3 \%$, of the datasets), gbm, bag, bstTree, blackboost and pcaNNet. In this group, extraTrees only achieves the best $R^{2}$ in 1 dataset, which represents $14.3 \%$, and achieves errors in $57.1 \%$ 


\begin{tabular}{|c||c|c|c||c|c||c|c||c|c|}
\hline & \multicolumn{3}{|c|}{$R^{2}$} & \multicolumn{2}{c|}{ RMSE } & \multicolumn{2}{c||}{ MAE } & \multicolumn{2}{c|}{ Best $R^{2}$} \\
\hline Pos. & Model & Rank & \%Error & Model & Rank & Model & Rank & Model & \%Best \\
\hline 1 & M5 & 6.57 & 0.0 & M5 & 6.57 & M5 & 5.14 & cubist & 42.9 \\
2 & cubist & 10.43 & 14.3 & cubist & 10.43 & cubist & 10.29 & extraTrees & 14.3 \\
3 & gbm & 11.43 & 0.0 & gbm & 11.43 & gbm & 12.43 & rf & 14.3 \\
4 & bag & 14.57 & 0.0 & bag & 14.57 & bag & 13.43 & brnn & 14.3 \\
5 & bstTree & 15.29 & 14.3 & bstTree & 15.29 & blackboost & 15.86 & dlkeras & 14.3 \\
6 & blackboost & 15.43 & 0.0 & blackboost & 15.43 & pcaNNet & 17.00 & - & - \\
7 & pcaNNet & 16.00 & 0.0 & pcaNNet & 15.71 & bstTree & 17.29 & - & - \\
8 & xgbTree & 19.57 & 14.3 & xgbTree & 19.43 & rlm & 20.00 & - & - \\
9 & lm & 21.43 & 0.0 & earth & 21.29 & xgbTree & 21.00 & - & - \\
10 & earth & 21.86 & 0.0 & kknn & 22.14 & kknn & 21.57 & - & - \\
\hline 11 & bayesglm & 21.86 & 0.0 & lm & 22.57 & dlkeras & 22.14 & - & - \\
12 & kknn & 22.14 & 14.3 & bayesglm & 22.57 & earth & 23.00 & - & - \\
13 & avNNet & 23.14 & 42.9 & avNNet & 23.14 & avNNet & 23.43 & - & - \\
14 & dlkeras & 23.43 & 14.3 & dlkeras & 23.71 & lm & 23.71 & - \\
15 & svr & 24.43 & 42.9 & lasso & 24.43 & svr & 25.29 & - \\
16 & lasso & 24.71 & 0.0 & svr & 24.71 & gam & 25.43 & - \\
17 & spikeslab & 25.29 & 0.0 & enet & 25.00 & bayesglm & 25.43 & - \\
18 & bagEarth & 25.57 & 14.3 & spikeslab & 25.29 & spikeslab & 25.43 & - & - \\
19 & enet & 26.14 & 14.3 & bagEarth & 25.43 & lasso & 25.71 & - & - \\
20 & gam & 26.14 & 0.0 & gam & 25.86 & lars & 26.14 & - & - \\
-
\end{tabular}

Table 12: List of the 20 best regression models according to the Friedman rank of $R^{2}$, RMSE and MAE for the 7 large-easy datasets (group LE).

of datasets. Since the datasets are easy, the $1 \mathrm{~m}$ also achieves a good position (9th). The models which fail in the 7 datasets of this group are kelm, wkpls, gprPol, krlsRad, rvmRadial, SBC, nodeHarvest and qrnn.

\subsection{Global discussion}

We also developed an analysis considering all the datasets together. Table 13 reports the 20 best models according to the Friedman rankings for $R^{2}$, RMSE and MAE over all the datasets, alongside with the percentage of datasets with errors for the 20 best models according to $R^{2}$ (column \%Error) and the percentage of datasets where each model achieves the best $R^{2}$ (column \%Best). The global results confirm the conclusions over the four dataset groups: cubist is globally the best regression model on the three rankings (although it achieves errors for $8.4 \%$ of datasets), followed by gbm 


\begin{tabular}{|c||c|c|c||c|c||c|c||c|c|}
\hline \multicolumn{1}{|c|}{} & \multicolumn{3}{|c|}{$R^{2}$} & \multicolumn{2}{c||}{ RMSE } & \multicolumn{2}{c|}{ MAE } & \multicolumn{2}{c|}{ Best $R^{2}$} \\
\hline Pos. & Model & Rank & \%Error & Model & Rank & Model & Rank & Model & $\%$ Best \\
\hline \hline 1 & cubist & 13.92 & 8.4 & cubist & 14.96 & cubist & 12.18 & extraTrees & 33.7 \\
2 & gbm & 15.42 & 1.2 & gbm & 15.34 & gbm & 16.12 & cubist & 15.7 \\
3 & bstTree & 15.84 & 6.0 & bstTree & 15.40 & bstTree & 16.23 & penalized & 8.4 \\
4 & M5 & 18.20 & 0.0 & M5 & 17.20 & M5 & 16.36 & bstTree & 6.0 \\
5 & avNNet & 19.23 & 14.5 & avNNet & 21.01 & avNNet & 20.16 & brnn & 4.8 \\
6 & extraTrees & 19.61 & 19.3 & bagEarth & 22.46 & qrf & 21.11 & avNNet & 3.6 \\
7 & qrf & 22.41 & 14.5 & bMachine & 22.48 & svr & 23.08 & rf & 3.6 \\
8 & pcaNNet & 23.49 & 0.0 & svr & 23.54 & extraTrees & 23.41 & bMachine & 3.6 \\
9 & rf & 23.82 & 24.1 & earth & 23.99 & bagEarth & 23.57 & dlkeras & 3.6 \\
10 & bMachine & 23.83 & 15.7 & blackboost & 24.39 & pcaNNet & 24.29 & gbm & 2.4 \\
11 & bagEarth & 24.14 & 7.2 & extraTrees & 24.71 & bMachine & 24.45 & M5 & 2.4 \\
12 & svr & 24.17 & 27.7 & pcaNNet & 24.83 & ppr & 24.76 & qrf & 2.4 \\
13 & ppr & 24.57 & 4.8 & ppr & 26.06 & kknn & 25.07 & bagEarth & 2.4 \\
14 & earth & 25.52 & 0.0 & kknn & 26.46 & earth & 25.40 & xgbTree & 2.4 \\
15 & blackboost & 25.69 & 0.0 & qrf & 26.84 & grnn & 25.92 & kelm & 1.2 \\
16 & kknn & 26.24 & 6.0 & rf & 27.01 & svmRad & 26.28 & ppr & 1.2 \\
17 & penalized & 27.70 & 12.0 & grnn & 27.37 & blackboost & 26.92 & kknn & 1.2 \\
18 & dlkeras & 28.07 & 7.2 & enet & 27.41 & bag & 27.27 & rbf & 1.2 \\
19 & svmRad & 29.14 & 28.9 & cforest & 27.53 & cforest & 27.28 & - & - \\
20 & grnn & 29.61 & 9.6 & bag & 27.64 & rf & 27.57 & - & - \\
\hline
\end{tabular}

Table 13: List of the 20 best models according to the Friedman rank of $R^{2}$, RMSE and MAE over all the datasets.

and bstTree. The difference is higher in terms of MAE (ranks 12.18 and 16.12 for cubist and gbm, respectively) than in terms of $R^{2}$ or RMSE. Cubist is also the second model which achieves more often the best $R^{2}$ (in $15.7 \%$ of datasets) after extraTrees (33.7\%), whose position is however much lower (6, 11 and 8 in the $R^{2}$, RMSE and MAE rankings, respectively), achieving errors for $19.3 \%$ of datasets. The M5 achieves position 4 in the three rankings, but it never fails, so its difference with cubist is caused by lower performance in datasets where cubist does not fail. Globally, the best neural network is avNNet (position 5). Other models in the top-10 of some rankings are qrf, pcanNet, rf (with $24.1 \%$ of errors), bMachine, bagEarth, svr $(27.7 \%$ of errors), earth and blackboost. Penalized, which is the best model for $8.4 \%$ of datasets, achieves position 17 in the $R^{2}$ ranking, with $12 \%$ of errors. 
372

The Im falls outside this table (positions 33-34).

\begin{tabular}{|c|c|c||c|c|c||c|c|c||c|c|c|}
\hline Pos. & Model & Rank & Pos. & Model & Rank & Pos. & Model & Rank & Pos. & Model & Rank \\
\hline \hline 1 & cubist & 11.1 & 6 & ppr & 19.5 & 11 & qrf & 22.1 & 16 & kknn & 24.8 \\
\hline 2 & gbm & 12.6 & 7 & pcaNNet & 20.4 & 12 & bMachine & 23.0 & 17 & rf & 26.0 \\
\hline 3 & M5 & 13.5 & 8 & earth & 20.6 & 13 & bagEarth & 23.6 & 18 & bag & 26.7 \\
\hline 4 & bstTree & 14.1 & 9 & blackboost & 21.0 & 14 & dlkeras & 23.9 & 19 & grnn & 28.0 \\
\hline 5 & avNNet & 18.8 & 10 & extraTrees & 21.1 & 15 & svr & 24.6 & 20 & cforest & 29.0 \\
\hline
\end{tabular}

Table 14: List of the 20 best regression models according to the Friedman rank of $R^{2}$ over all the datasets excepting PM2.5 Data 5 Chinese Cities.

Despite its high number of errors, extraTrees achieves a good position because it achieves the best $R^{2}$ for the majority of the thirteen PM2.5 Data 5 Chinese Cities datasets. In order to confirm that this fact does not bias the global results, we created an alternative ranking discarding these datasets (see Table 14). This alternative rank is rather similar to the previous one, being cubist, gbm, M5 and bstTree the first models, but extraTrees and rf move from positions 6 and 9 to 10 and 17, respectively.

We evaluated the statistical significance of the differences in $R^{2}$ among models with several tests. A Friedman test [74], implemented using the stats package, comparing all the models gives a $p$-value of $1.8 \cdot 10^{-45}<0.05$, which means that the difference among them is statistically significant. Table 15 reports the results of several statistical tests [75] developed to compare the globally best model (cubist) and the remaining 19 best models in terms of $R^{2}$. We used: 1) the paired-sample T-test, with the Matlab ttest (x,y) function: according to [75], since the number of datasets (83) is higher than 30 , the requirement of normal distributions for the $R^{2}$ values is not necessary; 2) the Dunnett's test [76] of multiple comparison, using the dunnet t8 Matlab

\footnotetext{
${ }^{8}$ https://es.mathworks.com/matlabcentral/fileexchange/38157-dunnett-m
} 


\begin{tabular}{|c|c|c|c|c|c|c|c|}
\hline Pos. & Model & Paired T & Dunnett & 2-Sample T & Wilcoxon & Sign & Post-Hoc \\
\hline \hline 2 & gbm & 0.825 & 1.000 & 0.921 & 0.593 & $0.001^{*}$ & 0.160 \\
\hline 3 & bstTree & 0.215 & 1.000 & 0.789 & 0.409 & $0.000^{*}$ & 0.974 \\
\hline 4 & M5 & 0.781 & 1.000 & 0.902 & 0.658 & $0.000^{*}$ & $0.007^{*}$ \\
\hline 5 & avNNet & $0.000^{*}$ & 0.451 & 0.068 & 0.075 & $0.000^{*}$ & $0.001^{*}$ \\
\hline 6 & extraTrees & $0.001^{*}$ & 0.474 & 0.083 & 0.124 & 0.909 & 0.671 \\
\hline 7 & qrf & $0.034^{*}$ & 0.995 & 0.383 & 0.285 & $0.006^{*}$ & $0.000^{*}$ \\
\hline 8 & pcaNNet & $0.034^{*}$ & 0.984 & 0.294 & 0.273 & $0.000^{*}$ & $0.042^{*}$ \\
\hline 9 & rf & $0.001^{*}$ & 0.375 & 0.064 & $0.048^{*}$ & $0.002^{*}$ & $0.000^{*}$ \\
\hline 10 & bMachine & $0.000^{*}$ & 0.624 & 0.109 & 0.051 & $0.000^{*}$ & $0.000^{*}$ \\
\hline 11 & bagEarth & $0.000^{*}$ & 0.518 & 0.074 & 0.086 & $0.000^{*}$ & $0.000^{*}$ \\
\hline 12 & svr & $0.000^{*}$ & $0.031^{*}$ & $0.005^{*}$ & $0.002^{*}$ & $0.000^{*}$ & 0.116 \\
\hline 13 & ppr & $0.004^{*}$ & 0.737 & 0.125 & 0.143 & $0.000^{*}$ & $0.000^{*}$ \\
\hline 14 & earth & $0.013^{*}$ & 0.919 & 0.204 & 0.188 & $0.000^{*}$ & $0.000^{*}$ \\
\hline 15 & blackboost & $0.045^{*}$ & 0.988 & 0.303 & 0.194 & $0.000^{*}$ & $0.000^{*}$ \\
\hline 16 & kknn & $0.003^{*}$ & 1.000 & 0.452 & 0.097 & $0.000^{*}$ & $0.000^{*}$ \\
\hline 17 & penalized & $0.000^{*}$ & $0.006^{*}$ & $0.000^{*}$ & $0.001^{*}$ & $0.000^{*}$ & $0.000^{*}$ \\
\hline 18 & dlkeras & $0.019^{*}$ & 0.992 & 0.343 & 0.133 & $0.000^{*}$ & $0.000^{*}$ \\
\hline 19 & svmRad & $0.000^{*}$ & $0.001^{*}$ & $0.000^{*}$ & $0.000^{*}$ & $0.000^{*}$ & $0.000^{*}$ \\
\hline 20 & grnn & $0.000^{*}$ & 0.897 & 0.196 & $0.037^{*}$ & $0.000^{*}$ & $0.000^{*}$ \\
\hline
\end{tabular}

Table 15: $p$-values achieved by the paired-sample T-test, Dunnett test, two-sample T-test, Wilcoxon ranksum test, sign test and Post-Hoc Friedman-Nemenyi test comparing the $R^{2}$ of the globally best model (cubist) and the remaining models in the top-20. The asterisks label models where the comparison is statistically significant $(p<0.05)$.

function; 3) the two-sample T-test, with the Matlab ttest2 function; 4) the Wilcoxon rank sum test [77], with the Matlab ranksum function; 5) the sign test, using the Matlab signtest function [77]; and 6) the Post-Hoc FriedmanNemenyi test (PMCMR [78] R package). The paired T-test gives significant differences, labeled as an asterisk $(*)$, except for the first three models, while the Dunnett, two-sample $\mathrm{T}$ and Wilcoxon tests only label few models as statistically different, including svr, penalized and svmRad (the Wilcoxon test also labels rf and grnn as different). The sign test, which counts the number of datasets where each regresor achieves the best $R^{2}$, labels all the models as statistically different to cubist excepting extraTrees. Finally, the 
Post-Hoc Friedman-Nemenyi test, which develops a comparison of multiple models, identifies as statistically significant the differences with all the models excepting gbm, bstTree extraTrees and svr.

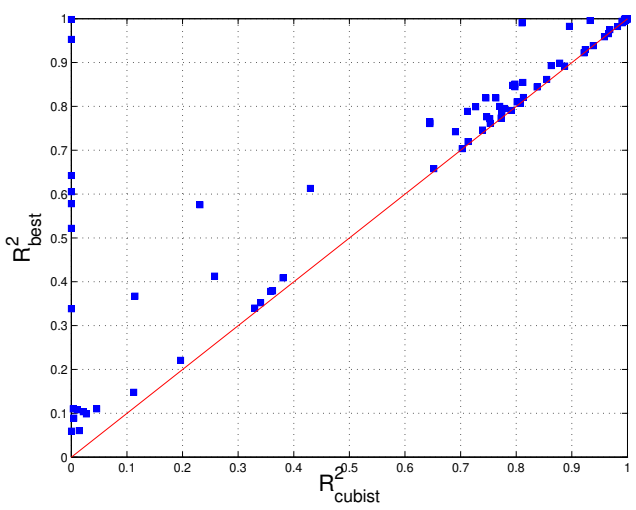

(a) Cubist.

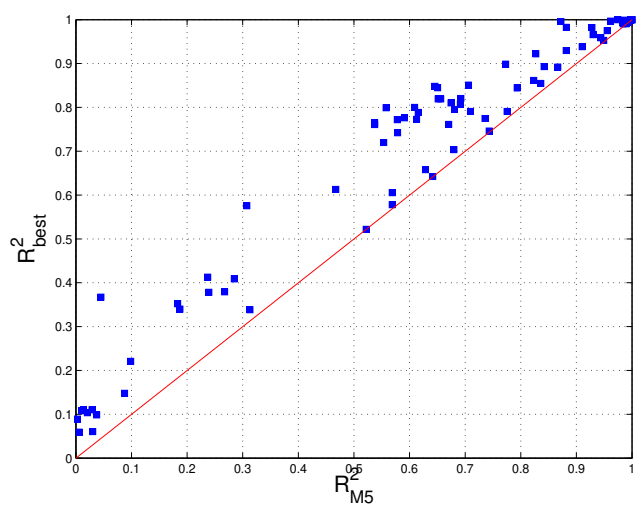

(b) M5.

Figure 2: Value of $R_{\text {best }}^{2}$ against $R^{2}$ for all the datasets.

Figures $2 \mathrm{a}$ and $2 \mathrm{~b}$ plot $R_{\text {best }}^{2}$ against $R_{\text {cubist }}^{2}$ and $R_{M 5}^{2}$, respectively, for all the datasets (M5 is the first regression model in the ranking which never fails). Cubist is near the best $R^{2}$ for all the points above 0.6, but its $R^{2}$ is almost zero for more than 10 points, due mainly to errors, which are on the vertical axis. However, all the points are near the red line for M5, which never fails, whose $R^{2}$ is near zero only for those datasets whose best $R^{2}$ is already almost zero, so the probability that M5 achieves $R^{2}$ near $R_{\text {best }}^{2}$ is much higher.

Left panel of Figure 3 plots the percentage of datasets where the difference $\Delta=R_{\text {best }}^{2}-R^{2}$ overcomes a threshold $\theta$, where $R^{2}$ is the value achieved by the first 4 models in Table 13; cubist, gbm, bstTree and M5. The model is better when the line is lower, because the percentage of datasets where $\Delta>\theta$ is lower. For low $\theta$ values, the lines follow the order cubist $<$ bstTree $<$ gbm $<$ M5, but the high error frequency of cubist and bstTree ( 7 and 5 , 

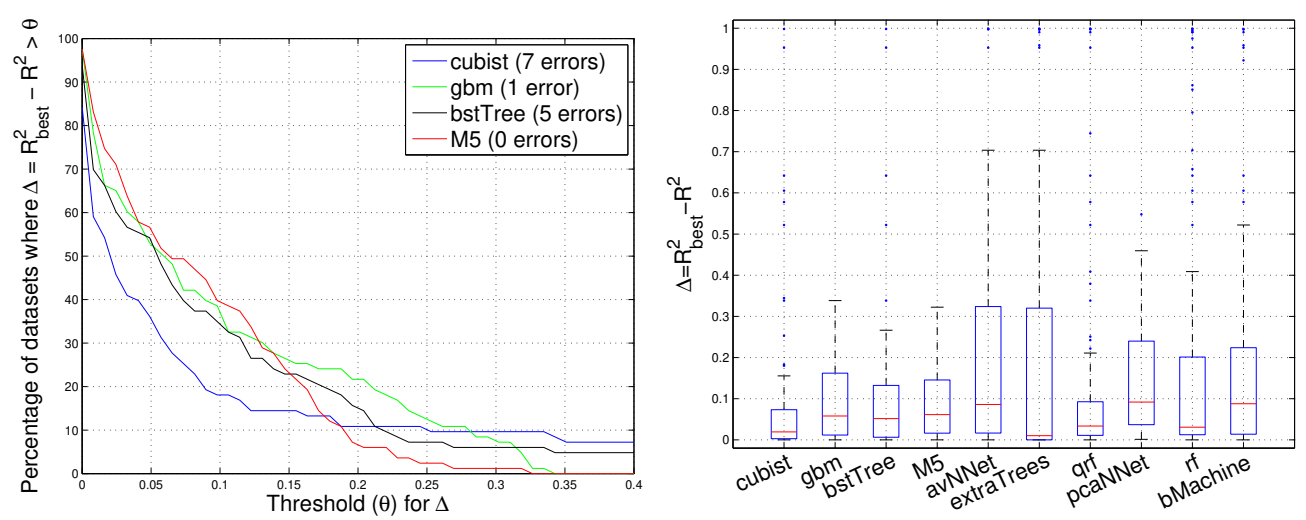

Figure 3: Left panel: percentage of datasets where the difference $\Delta=R_{\text {best }}^{2}-R^{2}$, with $R^{2}$ achieved by cubist, gbm, bstTree and M5, overcomes a given threshold $\theta$. Right panel: boxplots of the differences $R_{\text {best }}^{2}-R^{2}$ for the 10 best regressors.

respectively) causes that blue and black lines fall to zero for $\theta>0.4$ (outside the plot), while green and red lines (gbm and M5, respectively) fall to zero at 0.322 and 0.338 . Note that gbm fails (achieving $R^{2}=0$ ) only for dataset year-prediction, for which by chance $R_{\text {best }}^{2}$ is low (0.338), so $\Delta=0.338$ for this dataset and the green curve falls to zero at $\theta=0.338$. If the $R_{\text {best }}^{2}$ were higher, the green line would continue to the right without falling to zero, similarly to blue and black lines. The right panel of Figure 3 shows the boxplots of the differences $R_{\text {best }}^{2}-R^{2}$ for the first 10 models in the global ranking. The blue boxes report the $25 \%$ and $75 \%$ quantiles, while the red line inside the box is the median, and the blue points outside the box are the outliers. Although cubist, gbm, bstTree, extraTrees and rf exhibit low medians, all the models have several outliers (caused by datasets where they fail) with high $\Delta$ values, excepting M5, the only one which guarantees low $\Delta$ values (below 0.322) for all the datasets.

Figure 4 (left panel) plots $R_{\text {best }}^{2}$ and the $R^{2}$ achieved by M5 and gbm. M5 is 

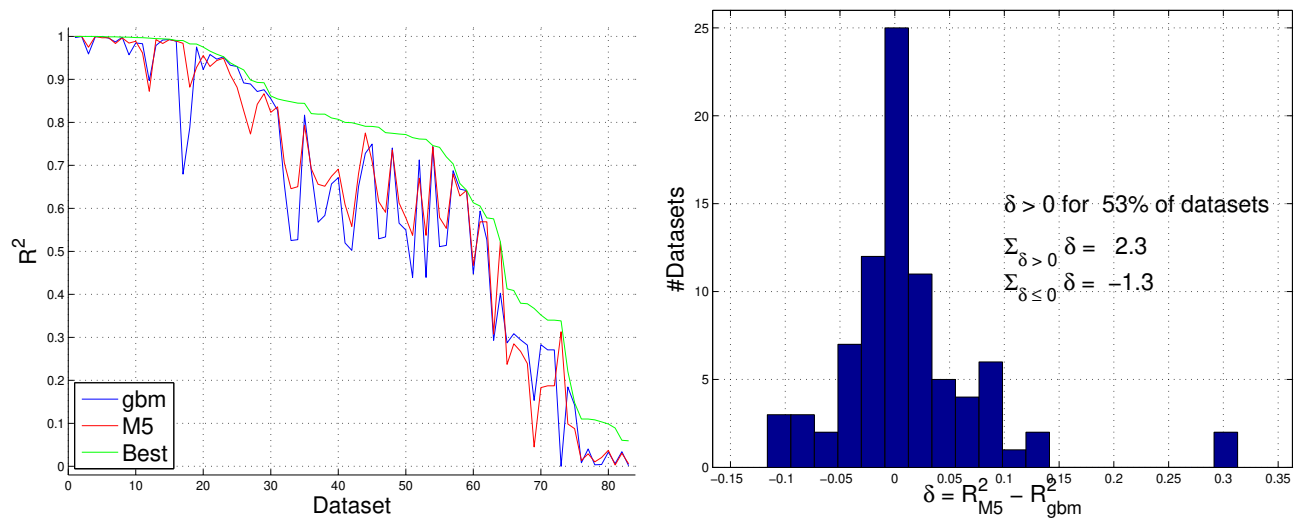

Figure 4: Left panel: $R_{g b m}^{2}$ (blue), $R_{M 5}^{2}$ (red) and $R_{\text {best }}^{2}$ (green) for each dataset, sorted by decreasing values of $R_{g b m}^{2}$ values. Right panel: histogram of the difference $R_{M 5}^{2}-R_{g b m}^{2}$.

near $R_{\text {best }}^{2}$ more often than gbm, and in several cases gbm is clearly below M5, but the former rarely outperforms the latter, and in these cases with lower difference. The right panel shows the histogram of the difference $R_{M 5}^{2}-R_{g b m}^{2}$ : its values are positive (i.e., M5 outperforms gbm) for $52.4 \%$ of the datasets, and when they are positive, they are higher (in absolute value) than when they are negative, so the sum of positive $\Delta$ values (2.3) outperforms the sum of negative values (-1.3). This shows that overall M5 outperforms gbm, although the latter is higher in the global ranking (Table 13). Remember that cubist, gbm and bstTree fail for some datasets, while M5 never fails.

In the left panel of Figure 4, the maximum difference $R_{\text {best }}^{2}-R_{M 5}^{2}$ is 0.322 in dataset student-mat, whose output is discrete with more than 10 values (see the left panel of Figure (5), so the dataset was not excluded. The low $R_{\text {best }}^{2}=0.3673$ for this dataset means that no model, and not only M5, fits accurately the true output, and both blue and green points fit equally bad the red line. The right panel of the same figure plots the difference $R_{\text {best }}^{2}-R_{M 5}^{2}$ against $R_{\text {best }}^{2}$. This difference is low for all the datasets (note that the vertical 

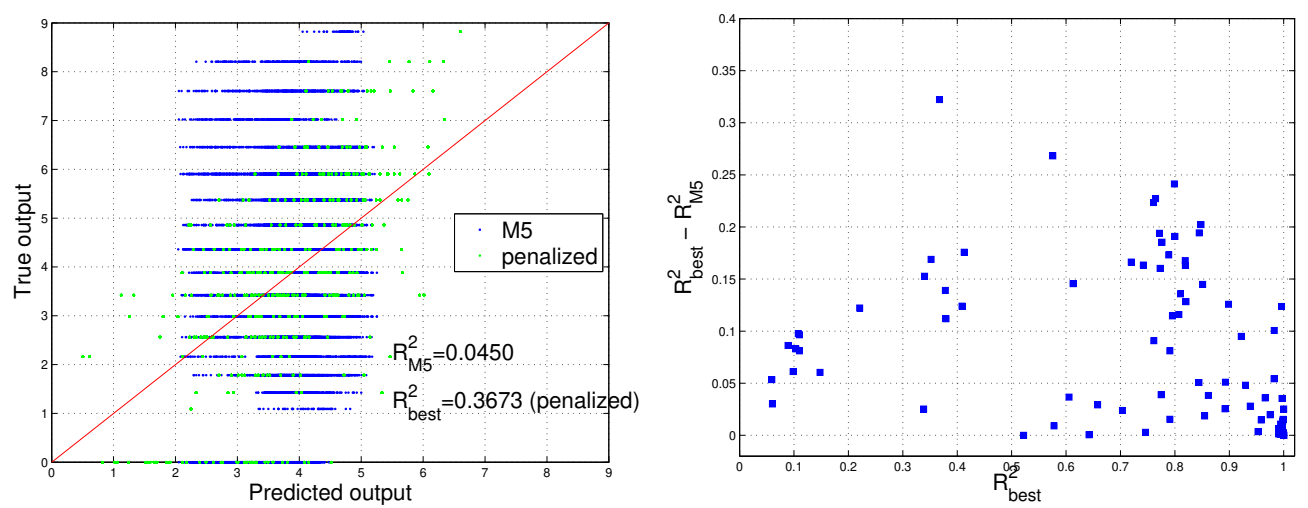

Figure 5: Left panel: true against predicted output for M5 (blue points) and the best regression model (penalized) for dataset student-mat. Right panel: difference $R_{b e s t}^{2}-R_{M 5}^{2}$ against $R_{\text {best }}^{2}$ for all the datasets.

scale is $0-0.4$ ), being below 0.2 (resp. 0.1) for $92.8 \%$ (resp. 60.2\%) of the datasets.
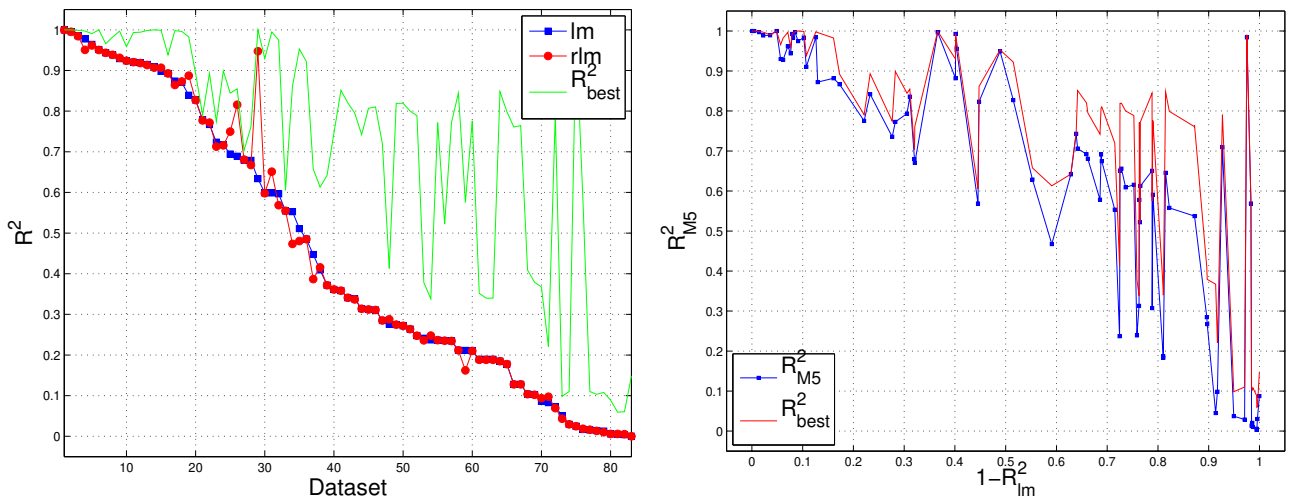

Figure 6: Left: values of $R_{l m}^{2}, R_{r l m}^{2}$ and $R_{\text {best }}^{2}$, sorted by decreasing $R_{l m}^{2}$. Right: $R_{M 5}^{2}$ and $R_{\text {best }}^{2}$ against $1-R_{l m}^{2}$.

ferc

overcomes 0.05 only in 6 datasets and its highest value is 0.31 , so that dataset 
outliers are few and not very relevant. In order to study the behavior of M5 with the dataset complexity, the right panel shows $R_{b e s t}^{2}$ and $R_{M 5}^{2}$ against $1-R_{l m}^{2}$, which measures the difficulty of the regression problem. The difference $R_{\text {best }}^{2}-R_{M 5}^{2}$, instead of raising with $1-R_{l m}^{2}$, achieves the highest values for $0.65<1-R_{l m}^{2}<0.9$. However, in the most difficult datasets, where $1-R_{l m}^{2}>0.9$, the $R_{M 5}^{2}$ follows very well $R_{b e s t}^{2}$, so M5 performs well even for hard datasets.
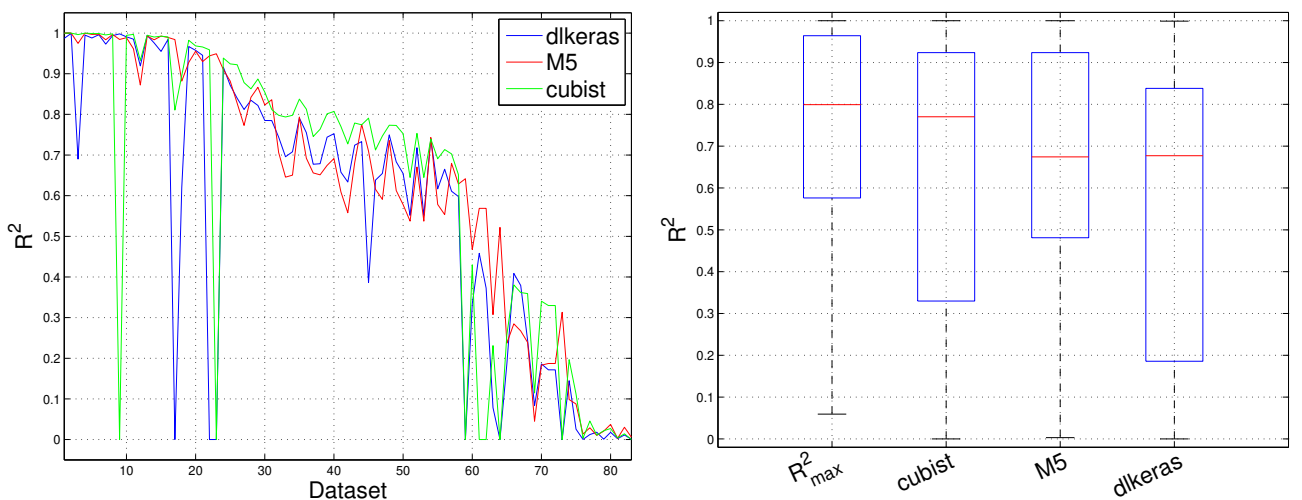

Figure 7: Left: values of $R^{2}$ achieved by dlkeras, M5 and cubist (datasets sorted by decreasing $\left.R_{\max }^{2}\right)$. Right: boxplots of $R_{\text {best }}^{2}$ and $R^{2}$ achieved by cubist, M5 and dlkeras over all the datasets.

Figure 7 (left panel) compares dlkeras to M5 and cubist over all the datasets (points with $R^{2}=0$ correspond to datasets where cubist or dlkeras fail). The cubist model (green line) achieves almost always the highest performance (in 61 of 83 datasets), while M5 overcomes cubist and dlkeras in 18 datasets, and dlkeras only in 4 datasets. In fact, cubist outperforms dlkeras in 71 datasets, while dlkeras outperforms cubist only in 8 datasets. The difference between dlkeras and M5 is lower (44 and 39 datasets favoring M5 and dlkeras, respectively. The right panel of Figure 7 shows the 
boxplots of $R_{\text {best }}^{2}$ and $R^{2}$ of cubist, M5 and dlkeras: this last box is clearly below cubist and M5, but its median is similar to M5 and lower than cubist. The upper box ends of cubist and M5 are near to $R_{b e s t}^{2}$, and the median of cubist is also very near to $R_{\text {best }}^{2}$, but the lower box ends of cubist and dlkeras are much below $R_{\text {best }}^{2}$ and M5 due to errors. Analyzing the parameter tuning of dlkeras, the largest available size $\left(75^{3}=421,875\right.$ neurons in three hidden layers) was selected only in 17 of 83 datasets. Therefore, in the remaining 66 datasets $R^{2}$ did not increase with larger networks, so they are not expected to provide better performances. However, they would spend higher computation times, overcoming the maximum allowed time (48 h.) more frequently, so dlkeras would achieve more errors than cubist and M5, which never fails.

\begin{tabular}{|l|c||l|c||l|l||l|c|}
\hline \multicolumn{2}{|c||}{ Small-difficult } & \multicolumn{2}{c||}{ Small-easy } & \multicolumn{2}{c|}{ Large-difficult } & \multicolumn{2}{c|}{ Large-easy } \\
\hline Family-model & Pos. & Family-model & Pos. & Family-model & Pos. & Family-model & Pos. \\
\hline \hline PLM-penalized & 1 & RGR-cubist & 1 & RGR-M5 & 1 & RGR-M5 & 1 \\
\hline RF-extraTrees & 2 & NET-avNNet & 2 & BST-gbm & 3 & BST-gbm & 3 \\
\hline NET-kelm & 4 & BST-bstTree & 3 & NN-kknn & 5 & BAG-bag & 4 \\
\hline BST-bstTree & 6 & BAG-bagEarth & 5 & DL-dlkeras & 8 & NET-pcaNNet & 7 \\
\hline SVR-svr & 8 & PRJ-ppr & 6 & SVR-svr & 9 & LR-lm & 9 \\
\hline SGP-gprRad & 10 & BYM-bMachine & 7 & NET-pcaNNet & 10 & AM-earth & 10 \\
\hline & & RF-extraTrees & 8 & & & & \\
\hline & & AM-earth & 9 & & & & \\
\hline
\end{tabular}

Table 16: Best model of each family within the 10 best positions in the $R^{2}$ Friedman ranking for each dataset group.

\subsection{Discussion by family of regression model}

It is interesting to analyze the behavior of the best model of each family. Table 16 reports the families with models in the top-10 of the $R^{2}$ ranking for each dataset group. The boosting family (BST, with models bstTree and 
gbm) and neural networks (NET, models kelm, avNNet and pcaNNet) families, are present in all the groups, while regression rules (RGR), with models cubist and M5, achieves the first position in three of four groups (small-easy, large-difficult, large-easy), and penalized linear regression (PLM) achieves the first position (penalized) in small-difficult datasets. Bagging (BAG, with models bag and bagEarth) and support vector regression (SVR, svr) are present in two groups, while RF (extraTrees), projection methods (PRJ, ppr), Gaussian processes (SGP, gprRad), nearest neighbors (NN, kknn), deep learning (DL, dlkeras) and linear regression (LR, lm and $\mathrm{rlm}$ ) are only present in just one group.

\begin{tabular}{|l|c|c||l|c|c|}
\hline Family & Best & Pos. & Family & Best & Pos. \\
\hline \hline Regression rules & cubist & 1 & Nearest neighbors & kknn & 16 \\
\hline Boosting & gbm & 2 & Penalized linear models & penalized & 17 \\
\hline Neural networks & avNNet & 5 & Deep learning & dlkeras & 18 \\
\hline Random forests & extraTrees & 6 & Ridge & foba & 27 \\
\hline Bayesian models & bMachine & 10 & Lasso & lars & 28 \\
\hline Bagging & bagEarth & 11 & Linear regression & lm & 33 \\
\hline Support vector regression & svr & 12 & Regression trees & ctree2 & 37 \\
\hline Projection methods & ppr & 13 & Gaussian processes & gprPol & 50 \\
\hline Generalized additive models & earth & 14 & Quantile regression & rqlasso & 56 \\
\hline
\end{tabular}

Table 17: Best regression model of each family and position in the global ranking.

Considering the global ranking, Table 17 reports the families, sorted by the position of their best models in Table 13. Only regression rules, boosting and neural networks are in the top-5, followed by random forests and Bayesian models with positions below 10. Most of the remaining families have best models which outperform $\operatorname{lm}$ (position 33), while regression trees, Gaussian processes and quantile regression achieve positions even higher. 


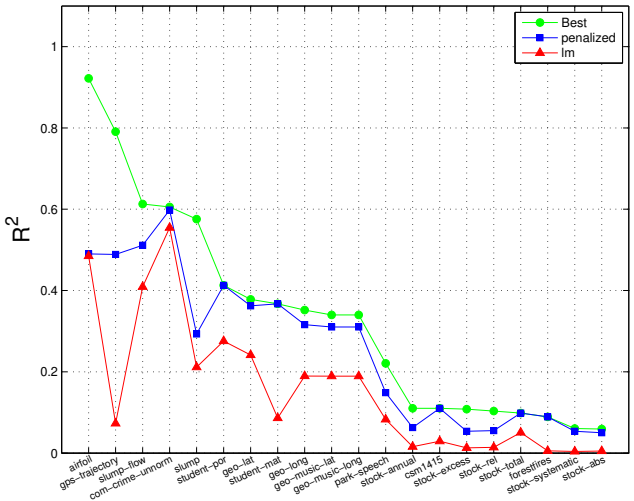

(a) Small-difficult (SD).

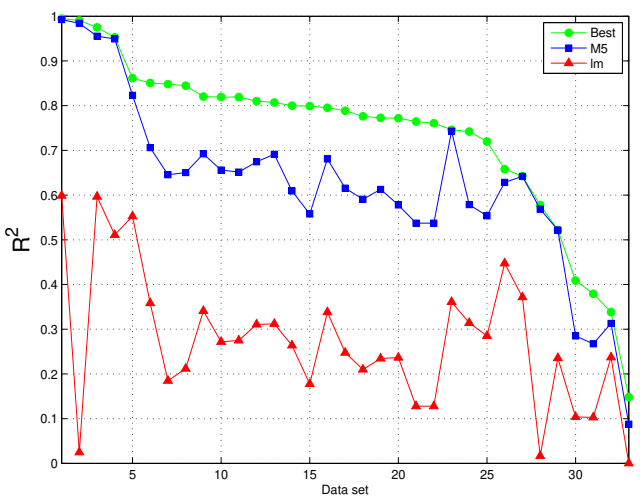

(c) Large-difficult (LD).

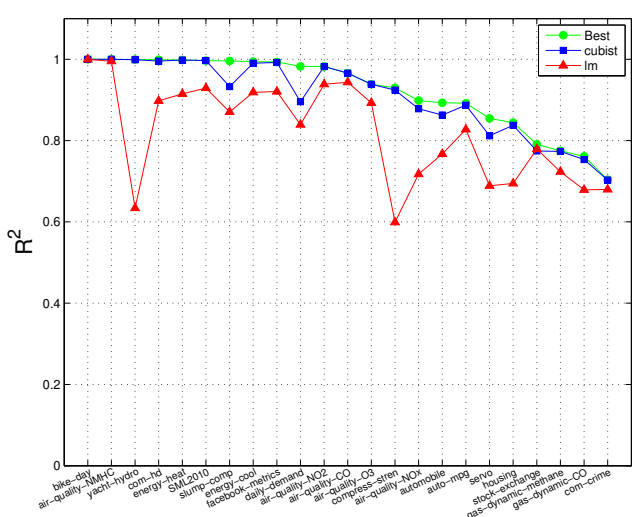

(b) Small-easy (SE).

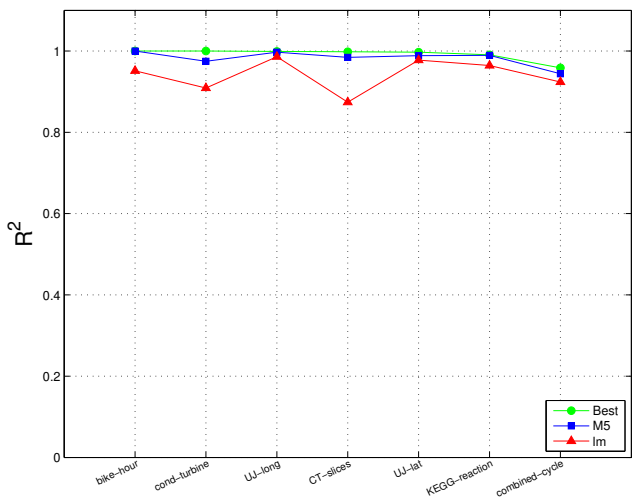

(d) Large-easy (LE).

Figure 8: Best $R^{2}$ (in green), $R^{2}$ achieved by the model with the best $R^{2}$ Friedman rank (in blue), and $R^{2}$ achieved by $1 \mathrm{~m}$ (in red) for each group. 


\subsection{Best result for each dataset}

The green line of Figure 4 shows $R_{\text {best }}^{2}$ for the 83 datasets. For 41 of them (which represents $49.39 \%$ ) the $R_{\text {best }}^{2}>0.8$, so that at least one model was able to predict the output with an acceptable accuracy (alternatively, these datasets might be considered as "easy" to learn). Besides, for other 20 datasets $\left(24.09 \%\right.$ of the total) the $R_{\text {best }}^{2}$ is between 0.6 and 0.8 , which is still an acceptable accuracy (datasets with middle difficulty). However, $R_{\text {best }}^{2}$ is between 0.2 and 0.6 for 13 datasets, which represents $15.66 \%$ (hard datasets), while $R_{\text {best }}^{2}<0.2$ for 9 datasets $(10.84 \%)$, where the models could not learn the regression problem at all. Some datasets are really hard, e.g. stock-abs, where $R_{\text {best }}^{2}=0.059$. Figure 8 plots the best $R^{2}$, alongside with $R^{2}$ achieved by the best model and by $1 \mathrm{~m}$ for each dataset group. In group $\mathrm{SD}$ (Figure 8a), the best model (penalized) is near to the best $R^{2}$ except for datasets airfoil, gps-trajectory, slump-flow and slump. Since this group includes small-difficult datasets, the $R^{2}$ of $1 \mathrm{~m}$ is always below 0.6 , but for the first five datasets some model achieves higher $R^{2}$ values. The penalized is also better than $1 \mathrm{~m}$ for all datasets, although the difference is low for datasets after geo-music-long. For group small-easy (SE, Figure $8 \mathrm{~b}$ ), the $R^{2}$ values of Im are higher, but the best model (cubist) is always very near to the best $R^{2}$ with some difference with respect to $1 \mathrm{~m}$. In the large-difficult group (LD, Figure $8 \mathrm{c}$ ), the $1 \mathrm{~m}$ values are very low and the best model (M5) is far from Im, following the best $R^{2}$ very closely for 12 of 33 datasets with a margin of 0.2-0.4 for the remaining 21 datasets. Finally, in group LE (large-easy datasets, Figure 8d ) the $1 \mathrm{~m}$ is already near the best $R^{2}$, although the best model (M5 again) always achieves the best $R^{2}$. 


\subsection{Discussion by elapsed times and memory consumption}

We studied the memory and time required by each regression model over all the datasets. Table 18 reports the information of the 20 best models according to the $R^{2}$ Friedman rank in each column: \%Best reports the percentage of datasets where they achieve the best $R^{2} ; \%$ Error reports the percentage of datasets where they failed; \%ME reports the percentage of memory errors caused by overcoming the largest allowed memory (128 GB); Datasets/mem reports the number of datasets run on the memory queues with $\left\{2^{i}\right\}_{i=1}^{6}$ GB.

In order to measure the time required by each model, the time spent in hyper-parameter tuning is discarded to avoid biasing caused by differences among models in the number of hyper-parameters and hyper-parameter values. The column \% TE reports the percentage of time errors, i.e., datasets where the model overcomes the maximum allowed time (48 h.). Although it may surprise that some models are not able to finish within $48 \mathrm{~h}$., we must consider the size of some datasets (more than 2 millions of patterns, up to 640 inputs) and the high number of trials (500) for some datasets. Generally, high values in the \%TE column happen with slow models, specially for large datasets. Since some models fail but do not overcome the allowed memory nor time, the sum of columns \% ME and \% TE is not always equal to column \%Error, e.g. nnls has no memory nor time errors, but \%Error is $4.8 \%$. The column Time reports the time (in sec.) spent by the model for a training+testing trial on dataset compress-stren, whose size might be considered "standard": 1,030 patterns and 8 inputs. The time is set to the maximum allowed time for models with errors in this dataset.

Comparing cubist, gbm, bstTree and M5 in terms of column \%Best, 


\begin{tabular}{|c|c|c|c|c|c|c|c|c|}
\hline & & & & & & \#Datasets/mem(GB) & \\
\hline Pos. & Model & Rank & \%Best & \%Error & \%ME & $2-4-8-16-32-64-128$ & \% TE & Time \\
\hline \hline 1 & cubist & 13.92 & 15.7 & 8.4 & & $68-6-8-1-0-0-0$ & 8.4 & 2.47 \\
\hline 2 & gbm & 15.42 & 2.4 & 1.2 & & $78-3-2-0-0-0-0$ & 1.2 & 1.46 \\
\hline 3 & bstTree & 15.84 & 6.0 & 6.0 & & $74-5-3-1-0-0-0$ & 6.0 & 3.84 \\
\hline 4 & M5 & 18.20 & 2.4 & & & $0-36-29-18-0-0-0$ & & 1.32 \\
\hline 5 & avNNet & 19.23 & 3.6 & 14.5 & & $77-3-2-0-0-1-0$ & 14.5 & 3.20 \\
\hline 6 & extraTrees & 19.61 & 33.7 & 19.3 & & $72-9-2-0-0-0-0$ & 20.5 & 3.62 \\
\hline 7 & qrf & 22.41 & 2.4 & 14.5 & & $62-12-4-1-3-1-0$ & 14.5 & 3.99 \\
\hline 8 & pcaNNet & 23.49 & & & & $77-3-3-0-0-0-0$ & & 1.36 \\
\hline 9 & rf & 23.82 & 3.6 & 24.1 & & $69-6-2-0-5-1-0$ & 24.1 & 3.05 \\
\hline 10 & bMachine & 23.83 & 3.6 & 15.7 & & $54-27-2-0-0-0-0$ & 16.8 & 12.10 \\
\hline 11 & bagEarth & 24.14 & 2.4 & 7.2 & & $76-1-4-1-1-0-0$ & 7.2 & 2.21 \\
\hline 12 & svr & 24.17 & & 27.7 & & $78-5-0-0-0-0-0$ & 27.7 & 172800 \\
\hline 13 & ppr & 24.57 & 1.2 & 4.8 & & $78-3-2-0-0-0-0$ & 4.8 & 1.24 \\
\hline 14 & earth & 25.52 & & & & $77-4-2-0-0-0-0$ & & 1.46 \\
\hline 15 & blackboost & 25.69 & & & & $75-1-3-0-3-0-1$ & & 1.48 \\
\hline 16 & kknn & 26.24 & 1.2 & 6.0 & & $80-2-1-0-0-0-0$ & 6.0 & 2.41 \\
\hline 17 & penalized & 27.70 & 8.4 & 12.0 & & $77-4-2-0-0-0-0$ & 12.0 & 1.54 \\
\hline 18 & dlkeras & 28.07 & 3.6 & 7.2 & & $83-0-0-0-0-0-0$ & 7.2 & 6.17 \\
\hline 19 & svmRad & 29.14 & & 28.9 & & $77-2-3-0-0-1-0$ & 28.9 & 172800 \\
\hline 20 & grnn & 29.61 & & 9.6 & 6.0 & $47-13-6-5-5-1-1$ & 3.6 & 0.22 \\
\hline \hline 28 & lars & 33.16 & & & & $79-2-2-0-0-0-0$ & & 0.03 \\
\hline 77 & qrnn & 77.00 & & 100.0 & & $77-4-2-0-0-0-0$ & 100.0 & 172800 \\
\hline \hline 63 & rndGLM & 51.80 & & 51.8 & 44.6 & $0-0-0-12-22-10-2$ & 7.2 & 2.54 \\
\hline
\end{tabular}

Table 18: List of the 20 first regression models sorted by increasing $R^{2}$ Friedman rank, with the percentage of datasets where each model achieves the best $R^{2}$ (column \%Best), percentage datasets with errors (column \%Error), percentage of memory errors (column $\% \mathrm{ME}$ ), number of datasets for each memory size (column \#Datasets/mem), percentage of time errors (\% TE) and training+test time (in sec.) spent for dataset compress-stren (column Time). Empty cells correspond to zero values.

cubist achieves often the best $R^{2}$ (15.7\% of datasets) followed by bstTree $(6 \%)$ while gbm and M5 tie (2.4\%). Cubist and bstTree fail in $8.4 \%$ and $6 \%$ of datasets, respectively, while gbm fails less (the three overcome the allowed time) and M5 never fails. None of them overcomes the memory limits, but M5 requires more memory (4-16 GB), while the others require 2-8 GB. Finally, M5 and gbm are faster (1.32 and $1.46 \mathrm{s./trial,} \mathrm{respectively),} \mathrm{while} \mathrm{bstTree}$ 
and cubist spend about 2-4 s. The avNNet and extraTrees spend about 3-4 s. but the former requires less memory (2 GB for 77 of 83 datasets) 9 . Among the remaining models, pcanNet never fails, is very fast $(1.36 \mathrm{~s}$.) and requires few memory (2 GB for 77 datasets), while bMachine is slower (12.1 s.) and requires more memory ( $4 \mathrm{~GB}$ for 27 datasets). The $\mathrm{rf}$ is faster (3.05 s.) with memory very variable with the dataset size (69 datasets with 2 GB but 1 with $64 \mathrm{~GB}$ ). On the other hand, svr and svmRad are are very slow with time errors in $28.9 \%$ of datasets, while grnn, ppr, earth and blackboost are fast (between 0.22 to 1.48 s.). However, grnn has time errors in $3.6 \%$ of datasets, requiring memory from 2 to 64 GB depending on the dataset with memory errors in $6 \%$ of datasets. Most models in positions 10-20 require few memory, and dlkeras requires the lowest memory (2 GB for all datasets), similar to kknn, although with time errors in $7.2 \%$ and $6 \%$ of datasets. To have time and memory references, the last three lines report the fastest and slowest models (lars and qrnn, respectively) in the compress-stren dataset, and the model which requires the largest memory (rndGLM). Considering times, lars spends $0.03 \mathrm{~s}$. being 26 times faster than M5 (the fastest model in the top-5), while qrnn is shutdown after $48 \mathrm{~h}$. in all the datasets, being 130,910 times slower than M5. With respect to memory, gbm and bstTree require only slightly more memory than dlkeras (2 GB for more than 74 datasets), while rndGLM always requires more than 16 GB with memory errors in $45.8 \%$ of the datasets.

Figure 9 (left panel) plots, in logarithmic scale, the times spent for each

\footnotetext{
${ }^{9}$ Both extraTrees and bartMachine use Java and by technical reasons their memory was limited to 8 GB.
} 

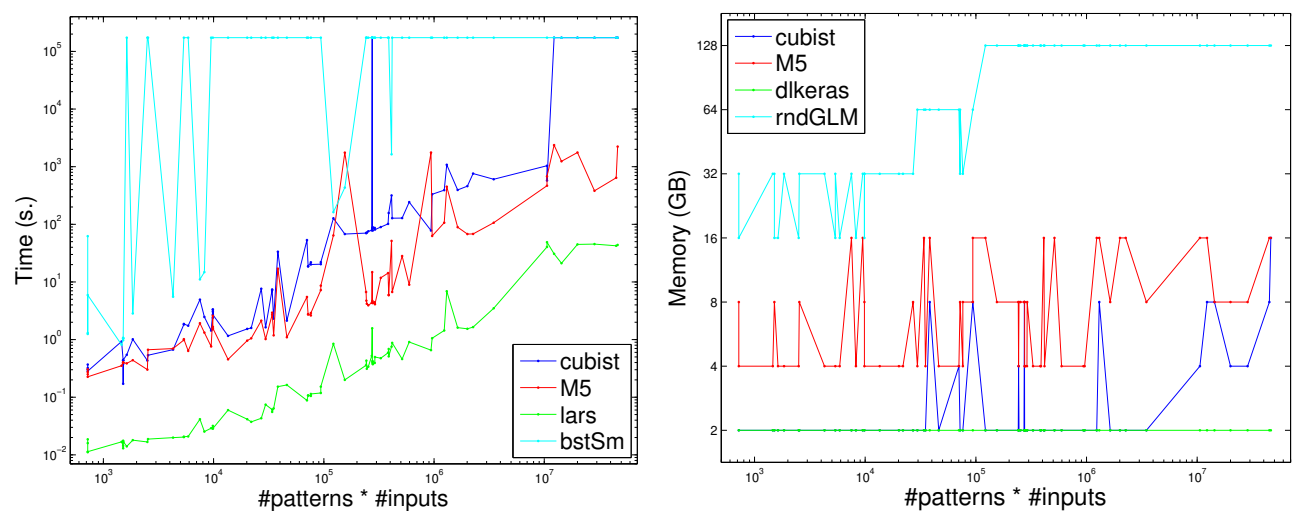

Figure 9: Left: times (in sec.) per trial spent by the best-performing (cubist and M5) and by the fastest and slowest regression models (lars and bstSm, respectively). Right: memory (in GB) required by cubist and M5, and by the models with least and most memory requirements (dlkeras and rndGLM, respectively). Both plotted against the product \#patterns·\#inputs.

dataset by cubist and M5, alongside with lars and bstSm, which are fastest and slowest models, respectively, for comparative purposes (qrnn is even slower than bstSm, but the former overcomes the allowed time in all the datasets so it is replaced by bstSm). The times are plotted against the product \#patterns.\#inputs of the dataset, which measures its size. Lars is one order of magnitude below M5 and cubist, which are similar for small datasets, but the difference grows with the dataset size. In largest datasets, cubist is almost two orders of magnitude slower than M5, overcoming the allowed time ( $48 \mathrm{~h}$. or $172,800 \mathrm{~s} . \sim 2 \cdot 10^{5}$ s.). Finally, bstSm is $2-3$ orders slower than lars for small datasets, but it already overcomes the time limit for some small, most medium and all large datasets (overall, for the $78.5 \%$ of datasets). Other slow models are xgbTree and xgbLinear, nodeHarvest, krlsRad and SBC, with average times between 20,000 and 300,000 s. and time errors for $50-85 \%$ of datasets. 
Considering memory, the right panel of Figure 9 plots cubist and M5, with dlkeras and rndGLM, which exhibit the lowest and highest memory requirements, against the product \#patterns·\#inputs. The dlkeras spends 2 GB for all the datasets, while cubist uses 2 GB excepting some medium and the 9 largest datasets. However, M5 requires more memory: 4, 8 and 16 GB for 36, 29 and 18 datasets, respectively. Comparatively, rndGLM requires 16, 32, 64 and $128 \mathrm{~GB}$ in 12, 22, 10 and 1 datasets, respectively, overcoming 128 GB in 39 datasets (45.8\%). Other models with high memory requirements are gprLin, gprPol and gprRad, rvmRad, krlsRad, wkpls, kelm and grnn, with memory errors in $6-10 \%$ of datasets.

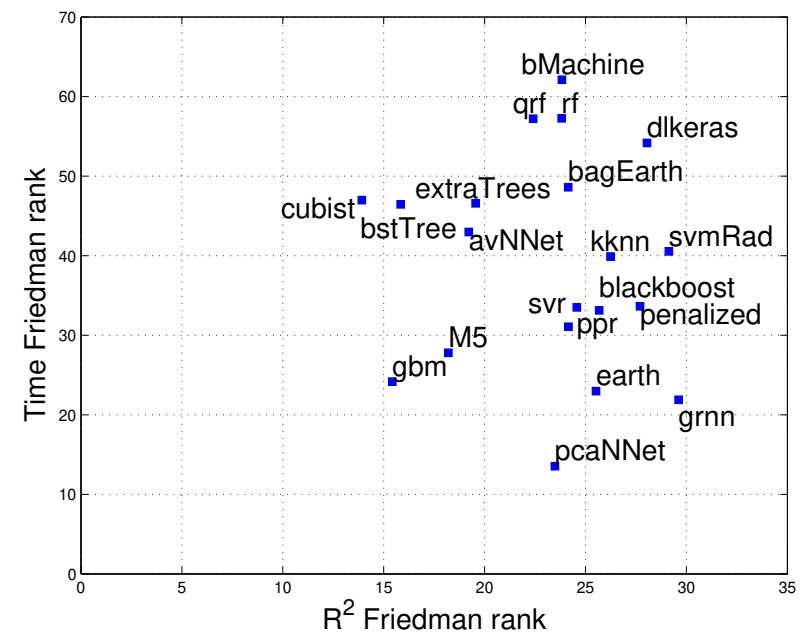

Figure 10: Friedman rank of the time (vertical axis) against the Friedman rank of $R^{2}$ (horizontal axis) for the 20 best models in Table 18.

Figure 10 plots the Friedman ranks of $R^{2}$ and time (horizontal and vertical axis, respectively) for the best 20 models. Cubist and pcaNNet achieve the lowest $R^{2}$ and time ranks, respectively, but the best trade-off between $R^{2}$ and time is achieved by gbm and M5. In fact, cubist is only slightly better 
than gbm according to $R^{2}$, but it is much slower. Other models with good $R^{2}$ are bstTree ( $R^{2}$ similar to gbm, but much slower), avNNet and extraTrees, but they are also slow. The following models according to $R^{2}$ rank are $\mathrm{rf}$, qrf, bMachine and bagEarth, whose $R^{2}$ rank is comparable to pcanNet but they are much slower. According to time, the models after pcanNet are grnn and earth, almost so fast as gbm but with much lower $R^{2}$.

\section{Conclusion}

The current work develops an exhaustive comparison of 77 regression methods, 73 implemented in $\mathrm{R}$ and other 4 in $\mathrm{C}++$, Matlab and Python, over the whole collection of 83 regression datasets of the UCI machine learning repository, including large datasets up to 2 millions of patterns and 640 inputs. The collection of regression models, that belong to 19 different families, aims to be a representative sample of the most popular and well-known methods currently available for regression tasks. The results have been evaluated in terms of $R^{2}$, RMSE and MAE, being similar with the three measurements, and depending on the dataset properties (size and difficulty, measured by the performance achieved by the classical linear regression). For small-difficult datasets, the penalized linear regression achieves the best results, followed by random forest (rf) and extremely randomized regression trees (extraTrees). For small-easy datasets, the M5 rule-based model with corrections based on nearest neighbors (cubist) achieves the best results, followed by the committee of back-propagation neural networks (avNNet) and the boosting ensemble of regression trees (bstTree). Finally, for both large-difficult and large-easy datasets the M5 regression tree is the best, fol- 
lowed the gradient boosted machine (gbm) and cubist. Considering globally all the datasets, cubist, gbm, bstTree and M5 achieve the best positions, and the differences between them are related mainly with: 1) the number of cases where they overcome the memory and time limits (128 GB and 48 h., respectively): cubist and bstTree fail in $8 \%$ and $6 \%$ of datasets, respectively, gbm only for $1 \%$ and M5 never fails; and 2) the speed (gbm, M5 and bstTree are 70, 30 and 10 times faster than cubist). In terms of $R^{2}$, gbm and M5 never decrease more than 0.35 below the best $R^{2}$ for any dataset, and $R_{\text {best }}^{2}-R_{M 5}^{2}>0.25$ only in $2.4 \%$ of datasets. Other models with good results are extremely randomized regression trees (extraTrees), which achieves the best $R^{2}$ in $33.7 \%$ of datasets, support vector regression (svr) and random forest ( $\mathrm{rf}$ ), but they are very slow, overcoming the maximum allowed time (48 h.) for more than $20 \%$ of the datasets. A post-hoc Friedman-Nemenyi test comparing cubist and the remaining models gives $p<0.05$ (i.e., difference statistically significant) excepting gbm, bstTree and extraTrees.

According to the position of their best regression models in the $R^{2}$ ranking, the best families are regression rules (whose best models are cubist and M5), boosting ensembles (gbm and bstTree), neural networks (avNNet), random forests (extraTrees and rf), projection methods (projection pursuit, ppr) and support vector regression (svr). Other families with models included in the top-20 are bagging ensembles (bagging ensemble of MARS models, bagEarth), generalized additive models (MARS, earth), nearest neighbors (kknn), generalized linear models (penalized) and deep learning (dlkeras). The remaining families exhibit poorer performances: ridge and LASSO, Bayesian models, linear regression, regression trees, Gaussian pro- 
cesses and quantile regression. The $R_{\text {best }}^{2}$ overcomes 0.5625 , considered the threshold for very good to excellent $R^{2}$ according to the Colton scale [79], for $76.2 \%$ of the datasets. Considering the elapsed time, the fastest model is least angle regression ( 1 ars), while M5 and cubist are 30 and 2,000 times slower, respectively. With respect to memory, the non-negative least squares regression (nnls) never requires more than 2 GB, while cubist and M5 require in average about 3 and $8 \mathrm{~GB}$, respectively, and the boosting ensemble of generalized linear models (rndGLM) requires about 78 GB, overcoming 128 GB in about half datasets. The future work includes to study the relations between the regression problem and the best models in order to predict the best model and its performance for a given dataset.

\section{Acknowledgment}

This work has received financial support from the Erasmus Mundus Euphrates programme [project number 2013-2540/001-001-EMA2], from the Xunta de Galicia (Centro singular de investigación de Galicia, accreditation 2016-2019) and the European Union (European Regional Development Fund - ERDF), Project MTM2016-76969-P (Spanish State Research Agency, AEI) co-funded by the European Regional Development Fund (ERDF) and IAP network from Belgian Science Policy.

\section{Appendix A. Dataset discrepancies with the UCI repository}

There are some discrepancies in Table 2 with respect to the original documentation of the UCI ML repository. Specifically, the beijing-pm25 dataset 
has 41,757 patterns, despite its description in the UCI documentation specifies 43,824 because 2067 patterns whose output is missing, so it can not be predicted, were removed. The cuff-less dataset has 73,200,000 patterns, while its description specifies 12,000. Instead of discarding it, we used the first 61,000 patterns. The greenhouse-net dataset has 2,921 files with 327 patterns per file, which gives 955,167 patterns instead of 2,921 in the dataset description. The household-consume dataset has 2,049,280 patterns instead of 2,075,259 as listed in the UCI documentation, because 25,979 original patterns have missing values (labeled as '?') for all the inputs and the output. The online-news dataset has 39,644 patterns instead of 39,797 as listed in the UCI documentation. For the UJIIndoorLoc datasets, output floor was discarded and did not give a separate regression dataset because it has only three different values.

\section{Appendix B. Listing of regression methods}

This appendix describes the regression model used in the current work, grouped by families, alongside with their software implementations and values of their tunable hyper-parameters. Default values are assumed for all the model parameters not cited explicitly.

\section{Linear regression (LR)}

1. $\mathbf{l m}$ is the linear regression model implemented by the stats package [11]. Collinear inputs exhibit undefined coefficients in the linear regression model returned by $\mathrm{lm}$, being discarded by it and by other models in the list, as we told above. 
2. rlm implements the robust linear model (MASS package), fitted using iteratively re-weighted least squares with maximum likelihood type estimation, which is robust to outliers in the output although not in inputs [13]. The only hyperparameter is the $\Psi$ function, which can be huber (Huber function, which leads to a convex optimization problem), hampel and Tukey bisquare, both with local minima. In our experiments, these functions are selected as the best $\Psi$ for $16 \%, 82 \%$ and $2 \%$, respectively, of the datasets.

\section{Penalized linear regression (PLM)}

3. penalized is the penalized linear regression (penalized package), which fits generalized linear models with a combination of L1 and L2 penalties. The L1 penalty, also named LASSO, penalizes the sum of absolute values of the coefficients, thus reducing the coefficients of inputs which are not relevant, similarly to input selection. The L2 penalty (also named ridge) penalizes the sum of squared coefficients, reducing the effects of input collinearity. The regression is regularized by weighting both penalties [15], whose weights are given by hyperparameters $\lambda_{1}$, tuned with values $1,2,4,8$ and 16 , and $\lambda_{2}$, with values $1,2,4$ and 8 . In our experiments, $\lambda_{1}=\lambda_{2}=1$ in the $87.9 \%$ of the datasets, and only in 10 of 83 datasets $\lambda_{1} \neq 1$ or $\lambda_{2} \neq 1$.

4. enet is the elastic-net regression model (elasticnet package), computed using the least angle regression - elasticnet (LARS-EN) algorithm [12]. Elastic-net provides a model for regularization and input selection, grouping together the inputs which are strongly correlated. This model is specially useful when the number of inputs is higher than 
the number of patterns, as opposed to LASSO models. There are two hyperparameters ( 5 values each one): the quadratic penalty, or regularization, hyperparameter $\left(\lambda\right.$, with values $\left.0,\left\{10^{-i}\right\}_{1}^{3}\right)$ and the fraction $\mathbf{s}$ of the L1 norm of the coefficient vector relative to the norm at the full least squares solution (the fraction mode is used in the predict.enet function, with values $0.05,0.28,0.52,0.76,1)$.

5. glmnet is the LASSO and elastic-net regularization for generalized linear models (GLM) implemented in the glmnet package [17]. The glmnet model uses penalized maximum likelihood to fit a GLM for the LASSO and elastic-net non-convex penalties. The mixing percentage $\alpha$ is tuned with 5 values from 0.1 to 1 : the value $\alpha=1$ (resp. $<1$ ) corresponds to the LASSO (resp. elastic-net) penalty. The selected value for $\alpha$ during hyperparameter tuning was 0.1 in $41.7 \%$ of the datasets. The regularization hyperparameter $\lambda$ is also tuned with values 0.00092 , 0.0092 and 0.092 .

6. glmSAIC is the generalized linear model with stepwise feature selection [19] using the Akaike information criterion and the stepAIC function in the MASS package (model glmStepAIC in the caret model list).

\section{Additive models (AM)}

7. gam is the generalized additive model (GAM) using splines (mgcv package). This model [21] is a GLM whose linear predictor is a sum of smooth functions (penalized regression splines) of the covariates. The estimation of the spline parameters uses the generalized cross validation criterion. The only hyperparameter is select, a boolean flag that 
adds an extra penalty term to each function penalizing its wiggliness (waving).

8. earth is the multivariate adaptive regression spline (MARS) in the earth package. This method [23] is a hybrid of GAM and regression trees (see family XII) which uses an expansion of product spline functions to model non-linear data and interactions among inputs. The spline number and parameters are automatically determined from the data using recursive partitioning, and distinguishing between additive contributions of each input and interactions among them. The functions are added iteratively to reduce maximally the residual, until its change is too small or a number of iterations is reached. The maximum number of terms in the model (nprune) is tuned with 15 values (less for some datasets) between 2 and 24 .

\section{Least squares (LS)}

9. nnls is the non-negative least squares regression (nnls package), which uses the Lawson-Hanson NNLS method [25] to solve for $\mathbf{x}$ the optimization problem $\min _{\mathbf{x}}|\mathbf{A x}-\mathbf{b}|$ subject to $\mathbf{x} \geq 0$, where $\mathbf{A}$ is the input data matrix, $\mathbf{b}$ is the true output and $\mathbf{x}$ is the linear predictor.

10. krlsRad is the radial basis function kernel regularized least squares regression (KRLS package), which uses Gaussian radial basis functions to learn the best fitting function which minimizes the squared loss of a Tikhonov regularization problem [27]. The KRLS method, which corresponds to the krlsRadial in the caret model list, learns a closed form function which is so interpretable as ordinary regression models. The only hyperparameter is the kernel spread $(\sigma)$, with 10 values in the 
set $\left\{10^{i}\right\}_{-7}^{2}$. By default, this method determines the trade-off between model fit and complexity, which is defined by the $\lambda$ parameter, by minimizing the sum of squared leave-one-out errors. The getModelInfo function only lists one value for $\lambda$, despite being listed as a tunable hyperparameter in the caret model list.

\section{Projection methods (PRJ)}

11. spls is the sparse partial least squares regression (spls package). This method [29] uses sparse linear combinations of the inputs in the dimensionality reduction of PLS in order to avoid lack of consistency of PLS with high dimensional patterns. The hyperparameters are the number of latent components $(\mathrm{K})$, with values 1, 2 and 3, and the threshold $(\eta)$, with 7 values from 0.1 to 0.9 .

12. simpls fits a PLS regression model with the simpls method [30], implemented by the plsr function in the pls package, with method=simpls. The PLS method projects the inputs and the output to a new space and it searches the direction in the input space which explains the maximum output variance. Simpls is particularly useful when there are more inputs than patterns and inputs are collinear. It directly calculates the PLS factors as linear combinations of the inputs maximizing a covariance criterion with orthogonality and normalization constraints. The only hyperparameter is the number of components (ncomp) used by the simpls model, with values from 1 to $\min (10$,\#inputs-1).

13. kpls is the PLS regression with method=kernelpls [31] in the same function and package as simpls, using the same hyperparameter setting as simpls with 6 values. This is the model named kernelpls in the 
caret model list.

14. wkpls uses method=widekernelpls [33] for PLS, tuning the number of components (ncomp) as simpls also with 10 values (model widekernelpls in the caret model list).

15. enpls.fs is an ensemble of sparse partial least squares (spls, see model \#12) regression models implemented by the enpls package [35]. The getModelInfo function lists only one value for the number of components (maxcomp), while the threshold argument, specified as a hyperparameter by the caret model list, is missing in the enpls.fit function.

16. plsRglm is the partial least squares generalized linear model (plsRglm package) with modele=pls-glm-gaussian [37]. The hyperparameters are the number of extracted components (nt), tuned with values 1,2 , 3 and 4, and the input significance level (alpha.pvals.expli), with values in the set $\left\{10^{i}\right\}_{-2}^{2}$.

17. ppr performs the projection pursuit regression (stats package), which models the output as a sum of averaging functions (mean, median, etc.) of linear combinations of the inputs [39]. The coefficients are iteratively calculated to minimize a projection pursuit (fitting criterion, given by the fraction of unexplained variance which is explained by each function) until it falls below a predefined threshold. The only tunable hyperparameter is the number of terms of the final model (nterms), with values from 1 to 10 .

18. pcr develops principal component regression (pls package), which models the output using classical linear regression with coefficients esti- 
mated with principal component analysis (PCA), i.e., using the principal components as inputs [41]. It works in three stages: 1) performs PCA and selects a subset of the principal components; 2) uses ordinary least squares to model the output vector using linear regression on the selected components; 3) uses the eigenvectors corresponding to the selected components in order to calculate the final pcr estimator transforming the modeled output vector to the original space, and estimates the regression coefficients for the original outputs. The number of components (ncomp) is tuned with values from 1 to $\min (10, \#$ inputs -1$)$.

19. icr is the independent component regression (caret package). The icr fits a linear regression model using independent component analysis (ICA), implemented by the fastICA package, instead of the original inputs [43]. The input data are considered a linear combination of a number of independent and non-Gaussian components (sources), so the training set matrix is written as the product of the source matrix and a linear mixed matrix, which contains the coefficients of the linear combination. The ICA estimates a "separating" matrix, which multiplied by the original data, provides the sources. This matrix must maximize the non-Gaussianity of the sources, measured by the neg-entropy. The only hyperparameter is the number of independent components n. comp, with values from 1 to min(10,\#inputs-1).

20. superpc is the supervised PCA (superpc package). This method [45] retains only a subset of the principal components which are correlated to the output. The tunable hyperparameters are the number of principal components (n.components), tuned with values 1, 2 and 3 (in all 
the datasets the value 1 is selected), and the threshold for retaining the input scores, with values 0.1 and 0.9 .

\section{Least absolute shrinkage and selection operator (LASSO) 10}

21. lasso performs LASSO regression, using the enet function in the elasticnet package with $\lambda=0$ to obtain the LASSO solution.

22. relaxo develops relaxed LASSO (relaxo package), which generalizes the LASSO shrinkage method for linear regression [14]. This method is designed to overcome the trade-off between speed and convergence in the L2-loss function of the regular LASSO, specially for sparse highdimensional patterns. It provides solutions sparser than LASSO with better prediction error. The relaxation hyperparameter $(\phi)$ is tuned with 7 values from 0.1 to 0.9 , while the penalty hyperparameter $(\lambda)$ is tuned with 3 data-dependent values.

23. lars is the least angle regression (lars package), a model selection method [16] which is less greedy than the typical forward selection methods. It starts with zero coefficients for all the inputs and finds the input $i$ most correlated with the output, increasing step-by-step its coefficient until another input $j$ has high correlation with the current residual (i.e., the error, or difference between the true and predicted outputs). The coefficients of inputs $i$ and $j$ are increased in the equiangular direction between inputs $i$ and $j$ until some other input $k$ is so correlated with the residual as input $j$. Then, it proceeds in the equiangular direction among $i, j$ and $k$, which is the "least angle direction",

\footnotetext{
${ }^{10}$ Due to the high number of models, LASSO models are included in a specific family and not in the penalized linear regression family.
} 
and so on until all the coefficients are non-zero (i.e., all the inputs are in the model). The lasso and fraction options are specified for training and prediction respectively, and the fraction hyperparameter (ratio between the L1 norm of the coefficient vector and the norm at the full LS solution) is tuned with 10 values between 0.05 and 1 (for $46.7 \%$ of datasets the selected value of fraction was 1 ).

\section{Ridge regression (RIDGE)11}

24. ridge develops ridge regression (elasticnet package), which introduces a regularization term, alongside with the squared difference between the desired and true outputs, in the function to optimize. This term, which evaluates the model complexity (e.g., the matrix norm for linear models), is weighted by the penalty or regularization hyperparameter $(\lambda)$. We use the enet function in the elasticnet package, already used for model enet, tuning $\lambda$ with 5 values between 0.01 and 0.1 (these two values are selected for $50 \%$ and $30 \%$ of the datasets, respectively).

25. spikeslab implements the spike and slab regression (spikeslab package), which computes weighted generalized ridge regression estimators using Bayesian spike and lab models [18]. The spikeslab method combines filtering for dimensionality reduction, model averaging using Bayesian model averaging, and variable selection using the gnet estimator. The only tunable hyperparameter is the number of selected inputs (vars), with the two values listed by the getModelInfo function: 2

\footnotetext{
${ }^{11}$ Similarly to LASSO, ridge regression models are grouped in a separate family instead of the penalized linear regression family.
} 
and the number of inputs (both selected with similar frequencies).

26. foba is the ridge regression with forward, backward and sparse input selection [20], implemented in the foba package. We use the adaptive forward-backward greedy version of the method (with the default value foba for the type argument of the foba function), which does a backward step when the ridge penalized risk increases in less than the parameter $\nu$ (with value 0.5 by default) multiplied by the ridge penalized risk reduction in the previous forward step. The hyperparameters are regularization for ridge regression $(\lambda)$, with 10 values between $10^{-5}$ and 0.1 , and the number of selected inputs or sparsity ( $\mathrm{k}$ ) for the prediction, with two values: 2 and the number of inputs.

VIII. Bayesian models (BYM)

27. bayesglm is the Bayesian GLM, implemented by the arm package. It uses expectation maximization to update the $\beta$ coefficients of the GLM at each iteration, using an augmented regression to represent the prior information [22]. The coefficients are calculated using a Student-t prior distribution.

28. brnn is the Bayesian regularized neural network (brnn package), a network with one hidden layer trained using Gauss-Newton optimization. The training minimizes a combination of squared error and a regularization term which uses the squared network weights [24]. The Bayesian regularization [80] determines the weights of both terms based on inference techniques. This requires an iterative computation of the Hessian matrix (or its Gauss-Newton approximation) of the performance with respect to the weights and biases until a goal is met or a maximum 
number of iterations is reached. The weights are not normalized, and the number of hidden neurons (neurons) is a hyperparameter tuned with values between 1 and 15, selecting neurons=1 in $31.6 \%$ of the datasets.

29. bMachine is the Bayesian additive regression tree (bartMachine package), which consists of a sum of regression trees and a regularization process developed on the parameters of the tree set [26]. It corresponds to bartMachine in the caret model list. We use the default number of trees (num_trees=50, the unique value listed by the getModelInfo function), and the tunable hyperparameters are the prior boundary (k), with values 2, 3 and 4 , and $\alpha$ (base value in tree prior to decide if a node is terminal or not), with 3 values between 0.9 and 0.99 .

\section{Space Gaussian processes (SGP, also known as kriging)}

30. gprLin implements Gaussian process regression (gaussprLinear in the caret model list), which interpolates values for the output using a sum of Gaussians, each specified by a mean and a covariance (or kernel) function that measures the similarity between inputs. This model uses linear (vanilladot) kernel in the gausspr function of the kernlab package.

31. gprRad (named gaussprRadial in the caret model list) uses the same function with Gaussian (rbfdot) kernel and automatically calculated kernel spread (default option kpar=1).

32. gprPol is the same method with polynomial (polydot) kernel (gausspr Poly in the caret model list), tuning the kernel hyperparameters degree, with values 1,2 and 3 , and scale, with values $\left\{10^{-i}\right\}_{1}^{3}$. 


\section{Quantile regression (QTR)}

33. rqlasso develops quantile regression with LASSO penalty, using the rq.lasso.fit function in the rqPen package. The quantile regression models optimize the so-called quantile regression error, which uses the tilted absolute value instead of the root mean squared error. This tilted function applies asymmetric weights to positive/negative errors, computing conditional quantiles of the predictive distribution. This method fits a quantile regression model with the LASSO penalty [32], tuning the regularization hyperparameter $\lambda$, with 10 values between 0.1 and $10^{-4}$ (for $76.7 \%$ of datasets the selected value was less than 0.01 ).

34. rqnc performs non-convex penalized quantile regression, with the rq. nc.fit function in the rqPen package. This model performs penalized quantile regression using local linear approximation [34] to maximize the penalized likelihood for non-convex penalties. The two hyperparameters are $\lambda$, with the same values as rqlasso, and penalty, which can be MCP (minimax concave penalty) or SCAD (smoothly clipped absolute deviation).

35. qrnn is the quantile regression neural network (qrnn package), a neural network which uses ramp transfer and quantile regression error functions [36]. The hyperparameters are number of hidden neurons (n.hidden), with 7 values from 1 to 13, and the penalty for weight decay regularization, with values $0,0.1$ and 0.0001 .

\section{Nearest neighbors (NN)}

36. kknn performs weighted k-nearest neighbors regression [38], implemented by the kknn package. The neighbors are weighted using a 
kernel function according to their distances to the test pattern. The only hyperparameter is the number of neighbors (kmax, with 10 odd values between 5 and 23).

\section{Regression trees (RGT)}

37. rpart is the classical regression tree trained using the recursive partitioning algorithm [40], implemented in the rpart package. Only the complexity hyperparameter (cp) is tuned (10 values).

38. nodeHarvest is a simple interpretable tree-based ensemble for highdimensional regression with sparse results [42] implemented in the nodeHarvest package. A starting tree of few thousand nodes is randomly generated. For a test pattern assigned to a node, the output is the mean of its training outputs; when the test pattern is assigned to several nodes, the output is the weighted average of their means. The selection of the nodes and their weights requires to solve a quadratic programming problem with linear inequality constraints. Only few nodes with non-zero weights are selected, so the solution is sparse. The hyperparameters are the maximal interaction depth (maxinter, with 10 values between 1 and 10, the most selected were 6-8) and the mode (2 values), which can be mean (weighted group means) or outbag (zero values in the smoothing matrix diagonal). This model is very slow, requiring huge times (more than 6 days) for high maxinter values and some datasets.

39. ctree 2 is the conditional inference tree (party package), which estimates the output using inference after a recursive partitioning of the input space [44]. The method tests the null hypothesis of statistical 
independence between any input and the output, and it stops when the hypothesis can not be rejected. Otherwise, it selects the input most related to the output, measured by the $p$-value of the partial test of independence between the output and that input. Then, it does a binary splitting of the selected input, and the two previous steps are recursively repeated. The hyperparameters are the threshold for $1-p$ in order to do a split (mincriterion), with 4 linearly spaced values between 0.01 and 0.99 , and the maximum tree depth (maxdepth), with integer values from 1 to 5 , selecting maxdepth $=5$ for $68.3 \%$ of datasets.

40. partDSA develops partitioning using deletion, substitution, and addition, implemented in the partDSA package [46]. This method recursively partitions the space considering that multiple inputs jointly influence the output, predicting a piecewise constant estimation through a parsimonious model of AND/OR conjunctions. The only hyperparameter is the maximum number of terminal partitions (cut.off.grow), tuned with integer values between 1 and 10, although the value 1 is selected for all the datasets. The parameter vfold is set to 1 in order to reduce the computational cost for large datasets.

41. evtree is the tree model from genetic algorithms [47] which uses evolutionary algorithms to learn globally optimal regression trees (evtree package). It chooses splits for the recursive partitioning in the forward stepwise search in order to optimize a global cost function. The only hyperparameter is the complexity $(\alpha)$ of the cost function, tuned with 10 linearly spaced values between 1 and 3, which weights negatively large tree sizes. 
${ }_{1022}$ XIII. Regression rules (RGR)

42. M5 is the model tree/rules [48] implemented in the RWeka package, tuning the flags pruned and smoothed (values yes/no each one), and rules/trees (to create a tree or a rule set) of the Weka M5 implementation.

43. cubist learns a M5 rule-based model with corrections based on nearest neighbors in the training set [50], implemented by the Cubist package. A tree structure is created and translated to a collection of rules, which are pruned and combined, and each rule gives a regression model, applied to the patterns which accomplish that rule. Cubist extends M5 with boosting when the hyperparameter committees $>1$, and using nearest neighbor based to correct the rule-based prediction. The tunable hyperparameters are the number of training committees (with 3 data-dependent odd values) and the number of neighbors (with values 0,5 and 9) for prediction.

44. SBC is the subtractive clustering and fuzzy C-means rules (frbs package), which uses substractive clustering to get the cluster centers of a fuzzy rule-based system for classification or regression [52]. Initially, each training pattern is weighted by a potential function which decreases with its distances to the remaining centers, and then it optimizes the centers using fuzzy C-means. The center with the highest potential is selected as a cluster center, and the potential of the remaining centers is updated. The only hyperparameter is the neighborhood radius (r.a), tuned with 7 linearly spaced values between 0 and 1 (this value is selected for nearly $50 \%$ of the 31 datasets where SBC does 
not fail). The selection of new cluster centers and potential updating is repeated until the potentials of the remaining patterns are below a pre-specified fraction of the potential of the first cluster center. Once all the centers are selected, they are optimized using fuzzy C-means. As we report in last rows of Table 4, we also tried the remaining 8 regression methods implemented in the frbs package and included in the caret model list (ANFIS, DENFIS, FIR.DM, GFS.FR.MOGUL, GFS.LT.RS, GFS.THRIFT, HYFIS and WM), but run-time errors happened for most or all the datasets.

\section{Random forests (RF)}

45. $\mathbf{r f}$ is the random forest ensemble of random regression trees implemented by the randomForest package [54]. The outputs of the base regression models are averaged to get the model output. Its only hyperparameter is the number of randomly selected inputs (mtry) with 10 linearly spaced values from 2 until the number of inputs, or less than 10 values when the number of dataset inputs is less than 11 (the lowest value mtry $=2$ was selected in $18 \%$ of the 64 datasets where $r f$ does not fail).

46. Boruta combines RF with feature selection (Boruta package). An input is removed when a statistical test proves that it is less relevant than a shadow random input, created by shuffling the original ones [55]. Conversely, inputs that are significantly better than shadowed ones are confirmed. The iterative search stops when only confirmed inputs are retained, or after a maximum number of iterations (maxRuns=100 by default), in which case non-confirmed inputs remain unless the iter- 
ations or the test $p$-value (0.01 by default) are increased. The only hyperparameter is mtry, tuned as in $r f$.

47. $\mathbf{R R F}$ is the regularized random forest (RRF package), which uses regularization for input selection in $\mathrm{rf}$, penalizing the selection of a new input for splitting when its Gini information gain is similar to the inputs included in the previous splits [57]. The hyperparameters are mtry, with 3 linearly spaced values between 2 and the number of inputs, and the regularization coefficient (coefReg), with values 0.01 and 1 , both selected with similar frequencies.

48. cforest is a forest ensemble of conditional inference trees [54], each one fitting one bootstrap sample (party package [58]). The only hyperparameter is the number of selected inputs (mtry, with values 2 and the number of inputs) of the conditional trees.

49. $\mathbf{q r f}$ is the quantile regression forest (quantregForest package [59]), a tree-based ensemble which generalizes RF in order to estimate conditional quantile functions. This regression model grows several RFs, storing all the training patterns associated to each node in each tree. For each test pattern, the weight of each training pattern is the average of the weights of all the training patterns in the leaves activated by that pattern in the different trees of the forest. Using these weights, the distribution function of each output value, and the conditional quantiles, are estimated. The only hyperparameter is mtry (tuned with 2 values as cforest). The quantile prediction threshold (argument what in the predict.quantregForest function) is set to 0.5 .

50. extraTrees is the ensemble of extremely randomized regression trees 
${ }_{1120}$ XVI. Boosting ensembles (BST)

XV. Bagging ensembles (BAG) trees. in the ipred package [64].

[60] implemented by the extraTrees package. It randomizes the input and cut-point of each split (or node in the tree), using a parameter which tunes the randomization strength. The full training set is used instead of a bootstrap replica. It is expected that explicit randomization of input and cut-point splittings combined with ensemble averaging should reduce the variance more than other methods. Its hyperparameters are the number of inputs randomly selected at each node (mtry, tuned with 2 values as cforest) and the minimum sample size to split a node (numRandomCuts), tuned with integer values from 1 to 10 (the selected value was 1 for $48.3 \%$ of the datasets).

51. bag [62] is the bagging ensemble of conditional inference regression trees (see model \#39) implemented by the caret package. The output for a test pattern is the average of the outputs over the base regression

52. bagEarth is the bagged MARS (caret package), a bagging ensemble of MARS base regression models implemented in the earth package (see model \#9), which learns a MARS model with degree=1 for each bootstrap sample. The only hyperparameter is the maximum number of terms (nprune) in the pruned regression model (10 values).

53. treebag is the bagged CART, a bagging ensemble of rpart regression base trees (see model \#37), implemented by the ipredbagg function

1121 54. rndGLM is a boosting ensemble of GLMs [65] implemented by the 
randomGLM package (also named randomGLM in the caret model list). It uses several bootstrap samples (nBags=100 by default) of the training set, randomly selecting inputs and interaction terms among them depending on the maxInteractionOrder hyperparameter, tuned with values 1, 2 and 3 (selected with frequencies $53.3 \%, 40 \%$ and $6.7 \%$, respectively). For each sample, inputs are ranked by its correlation with the output, and a predefined number of them are selected, using forward selection, to create a multivariate GLM. For a test pattern, the predicted value is the average of the GLM outputs. This regression model has very high memory requirements, overcoming the largest available memory (128GB) in 38 datasets, and requiring 128, 64, 32 and 16GB in 2, 10, 22 and 12 datasets, respectively.

55. BstLm is the gradient boosting machine with linear base models, implemented in the bst package. Gradient boosting optimizes arbitrary differentiable loss functions defining the fitting criteria [53]. Boosting combines weak base regression models into a strong ensemble by iteratively adding base models, and in each iteration the new model is trained to fit the error (residual) of the previous ensemble. Since the error can be viewed as the negative gradient of the squared error loss function, boosting can be considered a gradient descent method. BstLm uses the bst function with linear base models (argument learner=lm) and Gaussian family, since squared error loss is used. The only hyperparameter is the number of boosting iterations (mstop), with 10 values from 50 to 500 .

56. bstSm is the gradient boosting with smoothing spline base regression 
models (learner=sm in the bst function of the same package). The number of boosting iterations (mstop) is tuned with 10 values as BstLm.

57. bstTree is the gradient boosting with regression base trees (learner= tree, same function and package as BstLm). The hyperparameters are the number of boosting iterations (mstop, 4 values from 40 to 200) and the maximum depth of nodes in the final tree (maxdepth item in the list of the control.tree argument of the bst function), with integer values between 1 and 5 (this last value is selected in $55 \%$ of the datasets).

58. glmboost is the gradient boosting ensemble with GLM base regression models (glmboost function in the mboost package), tuning the number of boosting iterations (mstop, 10 values).

59. gamboost is the boosted generalized additive model (mboost package), a gradient boosting ensemble of GAM base regression models [69]. The ensemble minimizes a weighted sum of the loss function evaluated at the training patterns by computing its negative gradient. The base regression models are component-wise models (P-splines with a B-spline base, by default). The only hyperparameter is the number of initial boosting iterations (mstop), with 10 values from 50 to 500, selecting 500 as the best value for $56.7 \%$ of the datasets.

60. gbm is the generalized boosting regression model (gbm package [49]), named stochastic gradient boosting in the caret model list. The hyperparameters are the maximum depth of input interactions (interaction. depth), with integer values from 1 to 5 (the last value was selected in $48.3 \%$ of the datasets), and number of trees for prediction (n.trees), with values from 50 to 250 with step 50 (the value 250 was selected in 
${ }_{1193}$ XVII. Neural networks (NET) 0.1 (default values).

$45 \%$ of the datasets). We use a Gaussian distribution and shrinkage=

61. blackboost is the gradient boosting (blackboost function in the mboost package) with conditional inference regression base trees (ctree in the party package, see model \#40) and arbitrary loss functions [51]. The only hyperparameter is the maximum tree depth (maxdepth argument in the party: :ctree_control function, used as tree_controls argument of the blackboost function), with integer values from 1 to 5 , value selected in $79 \%$ of the datasets.

62. xgbTree is the extreme gradient boosting [53], using the xgb.train function in the xgboost package with booster=gbtree, root mean squared error as evaluation metric and linear regression as objective function. The hyperparameters are the maximum tree depth (max_depth), with values 1,2 and 3 (max_depth $=3$ for $53.3 \%$ of the datasets); maximum number of boosting iterations (nrounds), with values 50, 100 and 150 ; and learning rate $(\eta)$, with values 0.3 and 0.4 .

63. $\mathrm{xgbLinear}$ is the extreme gradient boosting with booster=gblinear and linear regression as objective function (xgboost package). Its hyperparameters are the L2 (square loss) regularization term on weights ( $\lambda$, with values $0,0.1$ and 0.0001$)$, bias ( $\alpha$, with values 0 and 0.1 ), and number of boosting iterations (nrounds, tuned as xgbTree).

64. mlpWD is the classical multi-layer perceptron with one hidden layer and weight decay (named mlpWeightDecay in the caret model list). It uses the mlp function in the RSNNS package, with learnFunc $=$ 
BackpropWeightDecay. The tunable hyperparameters are the size of the hidden layers (5 odd values between 1 and 5 ) and the weight decay (values 0, 0.1, 0.042, 0.01778 and 0.007498).

65. $\mathbf{m l p W D m l}$ is the same network with three hidden layers (RSNNS package, named mlpWeightDecayML in the caret model list), tuning four hyperparameters: the sizes of the three hidden layers (layer1, layer2 and layer3, tuned with values 1, 3 and 5 each one) and the weight decay (same values as mlpwD).

66. avNNet is the model averaged neural network (caret package). A committee of 5 (argument repeats) multi-layer perceptron neural networks of the same size trained using different random seeds, being averaged to give an output [81]. The boolean argument linout is set to have linear output neurons for regression, and MaxNWts is adjusted to allow the number of weights required by the dataset inputs. The hyperparameters are the network size, tuned with 7 odd values between 1 and 13, and the weight decay (with values $0,0.1$ and 0.0001 ).

67. $\mathbf{r b f}$ is the radial basis function network (RSNNS package) which does a linear combination of basis functions, each centered around a prototype [56]. The information is locally codified (opposed to globally in the MLP), the training should be faster and the network is more interpretable, although the output might be undefined if a test pattern does not activate any prototype. The only hyperparameter is the size of the hidden layer (10 odd values from 1 to 19$)$.

68. grnn is the generalized regression neural network [61], a special type of RBF network implemented by the Matlab neural network toolbox. 
After a clustering of the training set, the nodes of the hidden layer store the cluster centers, although the Matlab implementation uses so many clusters as training patterns. The output for a test pattern is a weighted sum of the Gaussian functions centered in the cluster centers, scaled by the cluster populations. During training, whenever a pattern is assigned to a cluster, the weight of the Gaussian function corresponding to that cluster is updated using the desired output. The Gaussian spread is the only hyperparameter (13 values between 0.01 and 2): large (resp. small) values lead to smooth (resp. close) approximations.

69. elm is the extreme learning machine [63] implemented by the elmNN package. The only hyperparameters are the number of hidden neurons (nhid), with 40 odd values between 1 and 79 (the last value was selected in $11.7 \%$ of the datasets), and the activation function (actfun), with 4 values: sin, radbas, purelin and tansig, selected with similar frequencies.

70. kelm is the ELM neural network with Gaussian kernel [63] using the publicly available Matlab code 12 . The hyperparameters are regularization $C$ and kernel spread $\sigma$, tuned with values $\left\{2^{i}\right\}_{-5}^{14}$ and $\left\{2^{i}\right\}_{-16}^{8}$, with 20 and 25 values, respectively.

71. pcaNNet is a multi-layer perceptron neural network with one hidden layer trained on the PCA-mapped training patterns, implemented by the caret and nnet packages. The principal components which account for more than $95 \%$ of the data variance are used for training. Each test pattern is mapped to the principal component space and the trained

\footnotetext{
${ }^{12}$ http://www.ntu.edu.sg/home/egbhuang/elm_kernel.html (visited March 29, 2017).
} 
${ }_{12 X} \mathrm{XVIII}$. Deep learning (DL)

pcanNet model gives an output. The tunable hyperparameters are the size of the hidden layer, with 7 values between 1 and 13, and the weight decay of the network, with values $0,0.1$ and 0.0001 .

72. bdk is the supervised bi-directional Kohonen network, implemented in the kohonen package [66]. The bdk combines Kohonen maps and counterpropagation networks using two maps, for inputs and output respectively. In each iteration, the direct (resp. inverse) pass updates only the weights of the input (resp. output) map, using a weighted similarity measurement (Euclidean distance for regression) which involves both maps, leading to a bi-directional updating. The test output is the weight of the winner node of the output map. The hyperparameters are the sizes of both maps (xdim and ydim, with 3 values from 3 to 17) and the initial weight (xweight) given to the input map during the distance calculation for the output map, and to the output map for updating the input map, tuned with values $0.5,0.75$ and 0.9 ).

73. dlkeras is the deep learning neural network implemented by the Keras module [67] of the Python programming language, with three hidden layers tuned with 50 and 75 neurons for each layer (nh1, nh2 and nh3, with 8 combinations). The deep learning methods [82, 83] are very popular, specially for image classification, and they are included in this comparison for regression tasks.

74. dnn is the deep belief network implemented in $\mathrm{R}$ by the DeepNet package [68]. It uses three hidden layers, tuning their number of neurons using 3 values for each layer (27 combinations). The weights are ini- 
tialized using stacked autoencoder (SAE), which in our experiments gave better results than deep belief network (DBN). Hidden and output neurons have hyperbolic tangent and linear activation functions, respectively.

\section{Support vector regression (SVR)}

75. $\mathbf{s v r}$ is the $\varepsilon$-support vector regression with Gaussian, accessed via the $\mathrm{C}++$ interface of the LibSVM library [9]. We tuned the regularization hyperparameter $C$ and the kernel spread $\gamma$ with values $\left\{2^{i}\right\}_{-5}^{14}$ and $\left\{2^{i}\right\}_{-16}^{8}$, with 20 and 25 values, respectively.

76. svmRad is another implementation of SVR (named svmRadial in the caret model list) with Gaussian kernel, which uses the (ksvm function in the kernlab package [70] for regression (argument type=eps-svr). This implementation also uses LibSVM, and it tunes the regularization hyperparameter $C$, with 20 values in the set $\left\{2^{i}\right\}_{-4}^{15}$, and the kernel spread $\sigma$. Although we specify 25 values for $\sigma$, the getModelInfo function only lists 6 values in the set $\left\{2^{-i}\right\}_{5}^{7}$.

77. rvmRad is the relevance vector machine [71] with Gaussian kernel (kernlab package), named rvmRadial in the caret model list. The RVM has the same functional form as the SVM, but it uses a Bayesian learning framework which reduces the number of basis functions, compared to the SVM, while keeping an accurate prediction. This regression model avoids the tunable regularization hyperparameter $(C)$ of the SVM, but it uses a method similar to expectation-maximization which, unlike SMO, may fall in local minima. The value of the Gaussian spread $\sigma$ is estimated by the getModelInfo function, which only 
lists one value.

\section{References}

[1] M. Fernández-Delgado, E. Cernadas, S. Barro, D. Amorim, Do we need hundreds of classifiers to solve real classification problems?, J. Mach. Learn. Res. 15 (2014) 3133-3181.

[2] D. H. Wolpert, The lack of a priori distinctions between learning algorithms, Neural Computation 9 (1996) 1341-1390.

[3] K. Bache, M. Lichman, UCI machine learning repository (2013). URL http://archive.ics.uci.edu/ml

[4] G. E. P. Box, D. R. Cox, An analysis of transformations, J. Royal Stat. Soc. Series B (Methodological) 26 (2) (1964) 211-252.

[5] R Team, R: A language and environment for statistical computing, Vienna, Austria, ISBN 3-900051-07-0 (2008).

URL https://www.R-project.org

[6] M. Kuhn, Caret: classification and regression training, R package (2016).

URL http://topepo.github.io/caret/train-models-by-tag.html

[7] Theano Team, Theano: A Python framework for fast computation of mathematical expressions, arXiv e-prints.

[8] Python Software Foundation, Python Language (2017).

URL https: //www . python .org 
[9] C. Chang, C. Lin, LIBSVM: a library for support vector machines, ACM Trans. on Intel. Syst. and Technol. 2 (2011) 27:1-27:27.

[10] Matlab, version 9.2 (R2017a), Natick (MA) (2011).

[11] J. Chambers, Linear models, J. M. Chambers and T. J. Hastie, Wadsworth \& Brooks/Cole, 1992, Ch. 4, pp. 96-138.

[12] H. Zou, T. Hastie, Regularization and variable selection via the elastic net, J. R. Stat. Soc. 67 (2005) 301-320.

[13] P. Huber, Robust statistics, Wiley, 1981.

[14] N. Meinshausen, Relaxed lasso, Comput. Stat. Data An. (2007) 374-393.

[15] J. Goeman, L-1 penalized estimation in the Cox proportional hazards model, Biometrical J. 52 (2010) 70-84.

[16] B. Efron, T. Hastie, I. Johnstone, R. Tibshirani, Least angle regression, Ann. Stat. 32 (2004) 407-499.

[17] N. Simon, J. Friedman, T. Hastie, R. Tibshirani, Regularization paths for Cox's proportional hazards model via coordinate descent, J. Stat. Softw. 39 (5) (2011) 1-13.

[18] H. Ishwaran, J. Rao, U. Kogalur, Spikeslab : prediction and variable selection using spike and slab regression, The R Journal 2 (2010) 68-73.

[19] B. Ripley, Modern applied statistics with S, Springer, 2002.

[20] T. Zhang, Adaptive forward-backward greedy algorithm for learning sparse representations, IEEE Trans. Inf. Theor. 57 (7) (2011) 4689-4708. 
[21] S. Wood, Fast stable restricted maximum likelihood and marginal likelihood estimation of semiparametric generalized linear models, J. Royal Stat. Soc. 1 (73) (2011) 3-36.

[22] A. Gelman, A. Jakulin, M. Pittau, Y. Su, A weakly informative default prior distribution for logistic and other regression models, Ann. Appl. Stat. 2 (4) (2009) 1360-1383.

[23] J. Friedman, Multivariate adaptive regression splines, Ann. Stat. 19 (1) (1991) 1-141.

[24] F. Foresee, M. T. Hagan, Gauss-Newton approximation to Bayesian regularization, in: Intl. Joint Conf. on Neural Netw., 1997, pp. 19301935.

[25] C. Lawson, R. Hanson, Solving least squares problems, Vol. 15 of Classics in Appl. Math., Soc. Ind. Appl. Math. (SIAM), 1995.

[26] A. Kapelner, J. Bleich, BartMachine: machine learning with Bayesian additive regression trees, J. Stat. Softw. 70 (4) (2016) 1-40.

[27] J. Hainmueller, C. Hazlett, Kernel regularized least squares: reducing misspecification bias with a flexible and interpretable machine learning approach, Polit. Anal. 22 (2013) 143-168.

[28] C. Willians, D. Barber, Ibayesian classification with gaussian processes, IEEE Trans. Pat. Anal. Mach. Intel. 20 (12) (1998) 1342-1351.

[29] H. Chun, S. Keles, Sparse partial least squares for simultaneous dimen- 
sion reduction and variable selection, J. of the Royal Stat. Soc. 72 (2010) 3-25.

[30] S. Jong, SIMPLS: an alternative approach to partial least squares regression, Chemometr. intel. lab. 18 (1993) 251-263.

[31] S. Jong, Comment on the PLS kernel algorithm, J. Chemometr. 8 (1994) $169-174$.

[32] I. Mizera, R. Koenker, Convex optimization in R, J. Stat. Softw. 60 (5) (2014) 1-23.

[33] S. Rännar, F. Lindgren, P. Geladi, S. Wold, A PLS kernel algorithm for data sets with many variables and fewer objects. part 1: theory and algorithm, J. Chemometr. 8 (1994) 111-125.

[34] H. Zou, R. Li, One-step sparse estimates in nonconcave penalized likelihood models, Ann. Stat. 36 (4) (2008) 1509-1533.

[35] N. Xiao, D. Cao, M. Li, Q. Xu, Enpls: an R package for ensemble partial least squares regression, arXiv preprint.

[36] A. Cannon, Quantile regression neural networks: implementation in $\mathrm{R}$ and application to precipitation downscaling, Comput. \& Geosci. 37 (2011) 1277-1284.

[37] F. Bertrand, M. Maumy-Bertrand, N. Meyer, Partial least squares regression for generalized linear models, r package version 1.1.1 (2014). URL http: //www-irma.u-strasbg.fr/ fbertran 
[38] K. Hechenbichler, K. Schliep, Weighted $k$-nearest-neighbor techniques and ordinal classification, Tech. rep., Ludwig-Maximilians University Munich (2004).

[39] J. Friedman, W. Stuetzle, Projection pursuit regression, J. Am. Stat. Assoc. 76 (1981) 817-823.

[40] L. Breiman, J. Friedman, R. Olshen, C. Stone, Classification and regression trees, Wadsworth and Brooks, 1984.

[41] B. Mevik, H. Cederkvist, Mean squared error of prediction (MSEP) estimates for principal component regression (PCR) and partial least squares regression (PLSR), J. Chemometr. 18 (9) (2004) 422-429.

[42] N. Meinshausen, Node harvest, Ann. Appl. Stat. 4 (4) (2010) 2049-2072.

[43] A. Hyvarinen, E. Oja, Independent component analysis: algorithms and applications, Neural networks 13 (2000) 411-430.

[44] T. Hothorn, K. Hornik, A. Zeileis, Unbiased recursive partitioning: a conditional inference framework, J. Comput. Graph. Stat. 15 (3) (2006) 651-674.

[45] E. Bair, R. Tibshirani, Semi-supervised methods to predict patient survival from gene expression data, PLoS Biol 2 (4) (2004) 511-522.

[46] A. Molinaro, K.Lostritto, M. van der Laan, PartDSA: deletion/substitution/ addition algorithm for partitioning the covariate space in prediction, Bioinformatics 26 (10) (2010) 1357-63. 
[47] T. Grubinger, A. Zeileis, K. Pfeiffer, Evtree: evolutionary learning of globally optimal classification and regression trees in R, J. Stat. Softw. 61 (1) (2014) 1-29.

[48] R. Quinlan, Learning with continuous classes, in: 5th Australian J. Conf. on Artif. Intel., 1992, pp. 343-348.

[49] G. Ridgeway, Gbm package, https://cran.r-project.org/package=gbm (2017).

[50] R. Quinlan, Combining instance-based and model-based learning, in: Proc. Intl. Conf. on Mach. Learn., 1993, pp. 236-243.

[51] P. Buehlmann, T. Hothorn, Boosting algorithms: regularization, prediction and model fitting (with discussion), Stat. Sci. 22 (4) (2007) 477-505.

[52] S. Chiu, Method and software for extracting fuzzy classification rules by subtractive clustering, in: Fuzzy Inf. Proc. Soc., NAFIPS, 1996, pp. 461-465.

[53] J. Friedman, Greedy function approximation: a gradient boosting machine, Ann. Stat. 29 (2001) 1189-1232.

[54] L. Breiman, Random forests, Mach. Learn. 45 (2001) 5-32.

[55] M. Kursa, W. Rudnicki, Feature selection with the Boruta package, J. Stat. Softw. 36 (11) (2010) 1-13.

[56] A. Zell et al., SNNS: Stuttgart Neural Network Simulator User Manual, Version 4.2, Tech. rep., IPVR, University of Stuttgart and WSI, University of Tbingen (1998). 
[57] H. Deng, G. Runger, Gene selection with guided regularized random forest, Pat. Recog. 46 (12) (2013) 3483-3489.

[58] T. Hothorn, Party package, http://cran.r-project.org/package=party (2018).

[59] N. Meinshausen, Quantile regression forests, J. Mach. Learn. Res. 7 (2006) 983-999.

[60] P. Geurts, D. Ernst, L. Wehenkel, Extremely randomized trees, Mach. Learn. 63 (1) (2006) 3-42.

[61] D. Specht, A general regression neural network, IEEE T. Neural Netw. 2 (1991) 568-576.

[62] L. Breiman, Bagging predictors, Mach. Learn. 24 (1996) 123-140.

[63] G.-B. Huang, H. Zhou, X. Ding, R. Zhang, Extreme learning machine for regression and multiclass classification, IEEE Trans. Systs., Man, and Cybern.-Part B: Cybern. 42(2) (2012) 513-529.

[64] A. Peters, Ipred package (2015). URL http://cran.r-project.org/package=ipred

[65] L. Song, P. Langfelder, S. Horvath, Random generalized linear model: a highly accurate and interpretable ensemble predictor, BMC Bioinformatics 14 (1) (2013) 1-22.

[66] W. Melssen, R. Wehrens, L. Buydens, Supervised Kohonen networks for classification problems, Chemom. Intell. Lab. Syst. 83 (2006) 99-113. 
[67] F. Chollet, Keras: The Python Deep Learning library (2015).

URL https://keras.io

[68] X. Ron, Deepnet package (2015).

URL https: //cran.r-project.org/package=deepnet

[69] P. Buehlmann, B. Yu, Boosting with the L2 loss: regression and classification, J. Am. Stat. Assoc. 98 (2003) 324-339.

[70] A. Karatzoglou, Kernlab package (2015).

URL https : //cran.r-project.org/package=kernlab

[71] M. Tipping, Sparse Bayesian learning and the relevance vector machine, J. Mach. Learn. Res. 1 (2001) 211-244.

[72] T. Hothorn, F. Leish, A. Zeileis, K. Hornik, The design and analysis of benchmark experiments, J. Comput. and Graph. Stat. 13 (3) (2005) 675-699.

[73] S. García, A. Fernández, A. Benítez, F. Herrera, Statistical comparisons by means of non-parametric tests: a case study on genetic based machine learning, in: Proc. II Congreso Español de Informática (CEDI 2007), 2007, pp. 95-104.

[74] M. Hollander, D. Wolfe, Nonparametric Statistical Methods, John Wiley \& Sons, 1973.

[75] J. Demšar, Statistical comparisons of classifiers over multiple data sets, J. Mach. Learn. Res. 7 (2006) 1-30. 
[76] C. Dunnett, A multiple comparison procedure for comparing several treatments with a control, J. Am. Stat. Assoc. 50 (1955) 1096-1121.

[77] J. D. Gibbons, S. Chakraborti, Nonparametric Statistical Inference, CRC Press, 2011.

[78] T. Pohlert, The pairwise multiple comparison of mean ranks package (PMCMR), r package (2014).

URL http://CRAN.R-project .org/package=PMCMR

[79] T. Colton, Statistical in medicine, Little Brown and Co., New York, NJ, 1974.

[80] D. MacKay, Bayesian interpolation, Neural Computation 4 (1992) 415447.

[81] B. Ripley, Pattern recognition and neural networks, Cambridge Univ. Press, 1996.

[82] G. E. Hinton, S. Osindero, Y.-W. Teh, A fast learning algorithm for deep belief nets, Neural Comput. 18 (7) (2006) 1527-1554.

[83] W. Liu, Z. Wang, X. Liu, N. Zeng, Y. Liu, F. Alsaadi, A survey of deep neural network architectures and their applications, Neurocomputing 234 (2017) 11-26. 